ARTICLE

https://doi.org/10.1038/s41467-019-09665-1

\title{
TET3 prevents terminal differentiation of adult NSCs by a non-catalytic action at Snrpn
}

\author{
Raquel Montalbán-Loro', Anna Lozano-Ureña ${ }^{1}$, Mitsuteru Ito (1) ${ }^{2}$, Christel Krueger ${ }^{3}$, Wolf Reik ${ }^{3,4}$, \\ Anne C. Ferguson-Smith ${ }^{2}$ \& Sacri R. Ferrón (1) ${ }^{1}$
}

Ten-eleven-translocation (TET) proteins catalyze DNA hydroxylation, playing an important role in demethylation of DNA in mammals. Remarkably, although hydroxymethylation levels are high in the mouse brain, the potential role of TET proteins in adult neurogenesis is unknown. We show here that a non-catalytic action of TET3 is essentially required for the maintenance of the neural stem cell (NSC) pool in the adult subventricular zone (SVZ) niche by preventing premature differentiation of NSCs into non-neurogenic astrocytes. This occurs through direct binding of TET3 to the paternal transcribed allele of the imprinted gene Small nuclear ribonucleoprotein-associated polypeptide N (Snrpn), contributing to transcriptional repression of the gene. The study also identifies BMP2 as an effector of the astrocytic terminal differentiation mediated by SNRPN. Our work describes a novel mechanism of control of an imprinted gene in the regulation of adult neurogenesis through an unconventional role of TET3.

\footnotetext{
${ }^{1}$ ERI BiotecMed/Departamento de Biología Celular, Universidad de Valencia, 46100 Valencia, Spain. ${ }^{2}$ Department of Genetics, University of Cambridge, Cambridge CB2 3EH, UK. ${ }^{3}$ Epigenetics Programme, The Babraham Institute, Cambridge CB22 3AT, UK. ${ }^{4}$ Wellcome Trust Sanger Institute, Cambridge CB10 1SA, UK. These authors contributed equally: Raquel Montalbán-Loro and Anna Lozano-Ureña. Correspondence and requests for materials should be addressed to S.R.F. (email: sacramento.rodriguez@uv.es)
} 
$\mathrm{n}$ the mammalian brain two regions generate new neurons throughout adulthood: the subventricular zone (SVZ) in the walls of the lateral ventricles and the subgranular zone (SGZ) of the dentate gyrus (DG) in the hippocampus ${ }^{1,2}$. The process of neurogenesis in these adult neurogenic niches is continually sustained by the activity of neural stem cells (NSCs) which are characterized by their ability to balance self-renewal with multipotential differentiation into astrocytes, oligodendrocytes, and neurons ${ }^{3}$. NSCs are lineage-related to radial glial cells, therefore, they exhibit astrocytic characteristics, such as glial fibrillary acidic protein (GFAP)+filaments or astrocyte-specific glutamate aspartate transporters (GLAST) ${ }^{4}$. NSCs also express the transcription factor Sox2 (Sry-related HMG box 2) ${ }^{4,5}$. In the SVZ in particular, NSCs also known as type B cells have a long basal process contacting blood vessels ${ }^{6}$ and extend an apical process ending in a primary cilium that protrudes into the ventricle ${ }^{7}$. The walls of the lateral ventricles thus show a typical organization where the apical process of type B cells are surrounded by a rosette of epithelial ependymal cells forming structures known as pinwheels ${ }^{8}$. Activated and quiescent NSCs appear to coexist in the $\mathrm{SVZ}^{9}$. Once activated, NSCs give rise to transit-amplifying progenitors or type C-cells, which in turn, generate neuroblasts, or type A-cells. These cells migrate anteriorly forming the rostral migratory stream (RMS) to the olfactory bulb (OB), where they mature into functional neurons involved in olfactory discrimination $^{10}$. Subventricular NSCs are also capable of producing some oligodendroblasts that migrate to the corpus callosum ${ }^{11,12}$.

Signaling between NSCs and other cells of their microenvironment or niche is critical for adult neurogenesis, through its interaction with NSCs intrinsic programs of gene expression ${ }^{9}$. Spatial and temporal gene regulation of gene expression in NSCs is established and maintained by the coordinated interaction between transcription factors and epigenetic regulators. Most mammalian genes are expressed from both maternally and paternally inherited chromosomal homologs. In contrast, imprinted genes are expressed from one parental copy only and the gene copy inherited from the other progenitor remains repressed $^{13}$. Their monoallelic expression makes these loci very vulnerable as mutation or deregulation of the sole expressed allele can compromise expression and lead to severe developmental defects $^{14,15}$. Interestingly, we have previously shown that selective absence of imprinting can occur in particular lineages to modulate the dosage of imprinted genes for cell-specific functions in physiological contexts ${ }^{16,17}$. These context-dependent changes may be important for cell plasticity during normal development and tissue regeneration ${ }^{18}$. Indeed, we have previously described that in the SVZ the paternally expressed gene Delta-like homolog 1 (Dlk1), an atypical Notch ligand, plays a relevant function in postnatal neurogenesis. Dlk1 is canonically imprinted elsewhere in the brain, however, it shows a selective absence of imprinting in subventricular NSCs with biallelic expression being required for stem cell maintenance and, ultimately, neurogenesis to the $\mathrm{OB}^{16}$. Another imprinted gene, the Insulin-like growth factor2 (Igf2), which is canonically expressed from the paternally inherited allele, is biallelically expressed in the choroid plexus resulting in a higher dose of IG2 secreted into the cerebrospinal fluid to regulate NSC proliferation ${ }^{17,19}$. Therefore, determining how imprinted genes operate in concert with signaling cues, as well as discovering the factors that modify methylation at imprinted regions in adult NSCs of different neurogenic niches, will lead to a better understanding of adult neurogenesis.

Genomic imprinting is regulated by epigenetic mechanisms, in particular DNA methylation imprints, that establish and maintain parental identity ${ }^{20}$. Epigenetic mechanisms are heritable modifications to DNA and chromatin, that do not involve changes in the DNA sequence itself, but which can modulate gene expression and genome function ${ }^{21}$. At the molecular level, epigenetic regulation involves chemical modifications to DNA such as methylation and hydroxymethylation, and to the histone proteins around which the DNA is wrapped, having a relevant role in neurogenesis 22 . Ten-eleven translocation (TET) family of enzymes have been found to play an important role in passive replication-dependent and active demethylation of DNA in mammals ${ }^{23}$. These processes can involve the 5hydroxymethylcytosine $(5 \mathrm{hmC}), 5$-formylcytosine $(5 \mathrm{fC})$, and 5 -carboxylcytosine $(5 \mathrm{caC})$ intermediates that are produced by the oxidation of 5 -methylcytosine $(5 \mathrm{mC})^{24,25}$. In mammals, three members of the TET family have been identified: TET1, TET2, and TET $3^{24}$. Loss of TET enzymes revealed promoter hypermethylation and deregulation of genes implicated in embryonic development and differentiation ${ }^{26}$. Specifically, elimination of Tet 3 results in defects in neural progenitor proliferation and differentiation ${ }^{27-29}$. In line with this, while Tet3 knockout ESCs can be successfully induced to neural progenitors, they undergo rapid apoptosis and show greatly compromised terminal differentiation ${ }^{30}$.

TET proteins have been implicated in maintaining DNA methylation at imprinted regions during the germline resetting of genomic imprints and in embryonic stem cells ${ }^{31-33}$. However, whether TET proteins can also be involved in the modulation of DNA imprints in adult NSCs has not been established. In addition to the well-described catalytic activity of TET proteins, the preferential binding of TET proteins at $5 \mathrm{mC}$-free promoters and their interaction with histone deacetylases, acetyltransferases, Polycomb repressive complex 2, and O-linked $\mathrm{N}$-acetylglucosamine transferase to regulate gene expression ${ }^{34,35}$, suggest additional potential functions independent of its catalytic activity ${ }^{36}$. This possibility is supported by the demonstration that non-catalytic forms of TET are able to rescue the proliferative phenotype of Tet2 knockout cells ${ }^{37}$. Moreover, overexpression of TET1 or a catalytically inactive mutant in the mouse hippocampus results in the upregulation of several neuronal memory-associated genes ${ }^{38}$, further supporting nonconventional functions of TET proteins.

There are many intriguing questions concerning the role of genomic imprinting and gene dosage during the neurogenesis process. Here we demonstrate a function for the non-catalytic form of TET3 in the maintenance of adult NSC state by preventing the differentiation of type $\mathrm{B}$ cells into non-neurogenic astrocytes. The mechanism involves direct binding and repression of TET3 to the imprinted gene Snrpn. We also show that astrocytic differentiation of adult NSCs in response to Snrpn deregulation involves increased production of BMP2. Our study provides important new insights into the role of TET3 in neurogenesis and emphasizes the importance of correct regulation of imprinted genes in the neurogenic niche.

\section{Results}

Tet3 deficiency causes depletion of the NSC pool in vivo. TET dioxygenases convert $5 \mathrm{mC}$ to $5 \mathrm{hmC}$ resulting in the removal of the methylated cytosine in somatic tissues, including the brain $^{39-41}$. Quantitative RT-PCR (qPCR) assays showed that Tet2 and Tet 3 were the most abundant members of the Tet dioxygenases in the adult SVZ, being also highly expressed in NSCs isolated from the early postnatal SVZ (Fig. 1a). However, only Tet 3 expression was maintained postnatally in the stem cell pool and in differentiated cells (Fig. 1a). Immunofluorescent analyses with antibodies to TET3 and to cell-identity antigens in wildtype adult brains revealed nuclear staining for TET3 protein in the GFAP/SOX2+population located close to the lateral ventricles (Fig. 1b), whereas no expression was observed in 
a

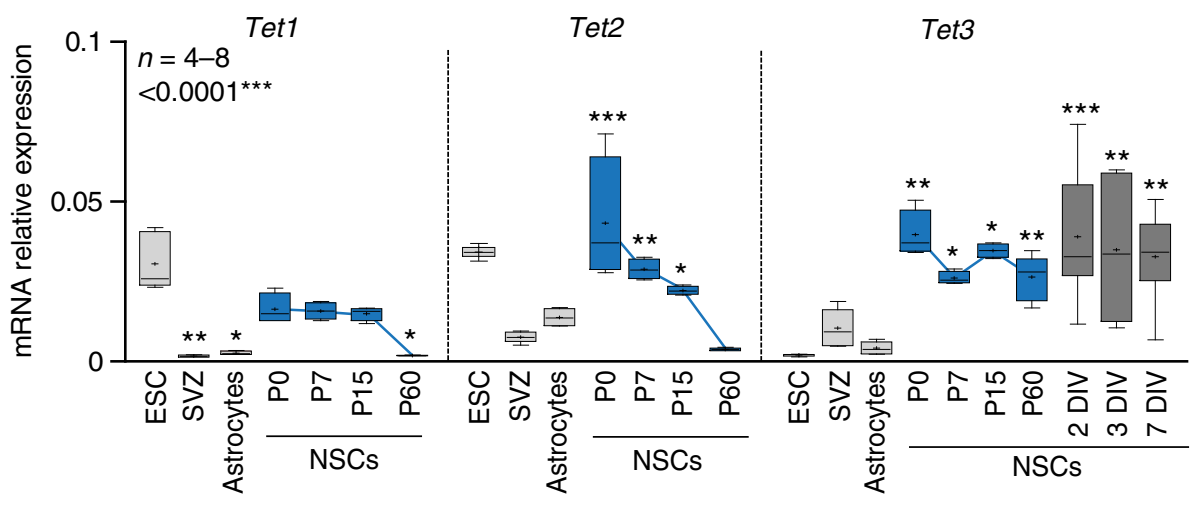

b
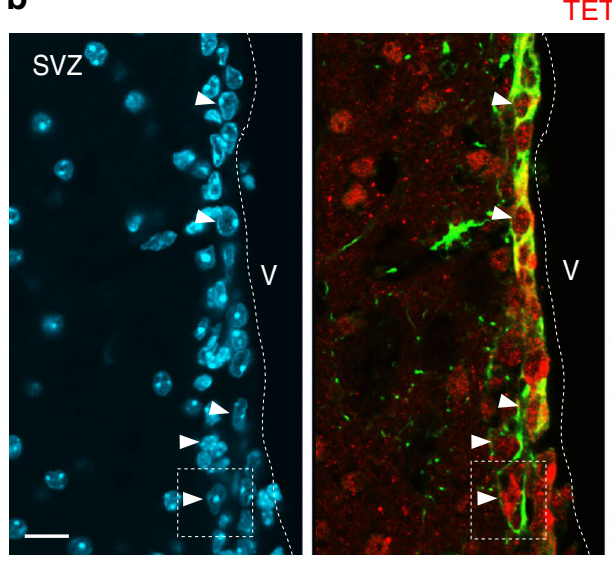

ET3 GFAP SOX2 DAPI

C

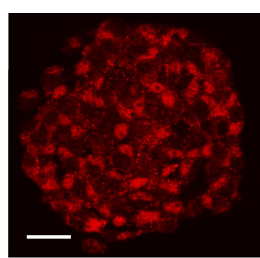

TET3

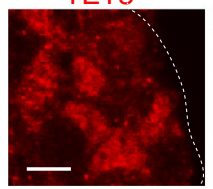

TET3 SOX2 DAPI

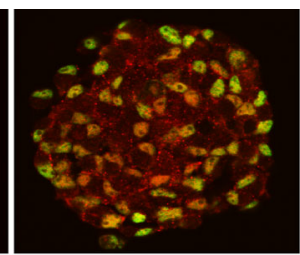

SOX2

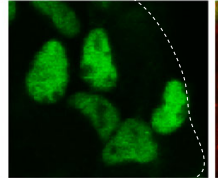

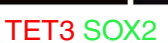

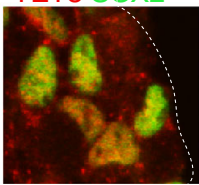

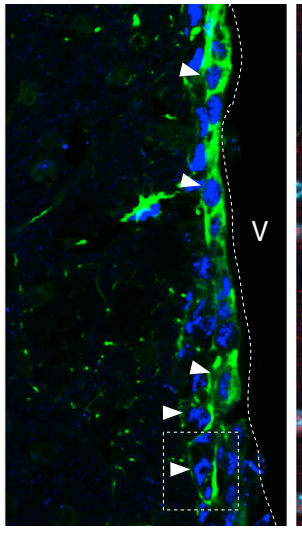
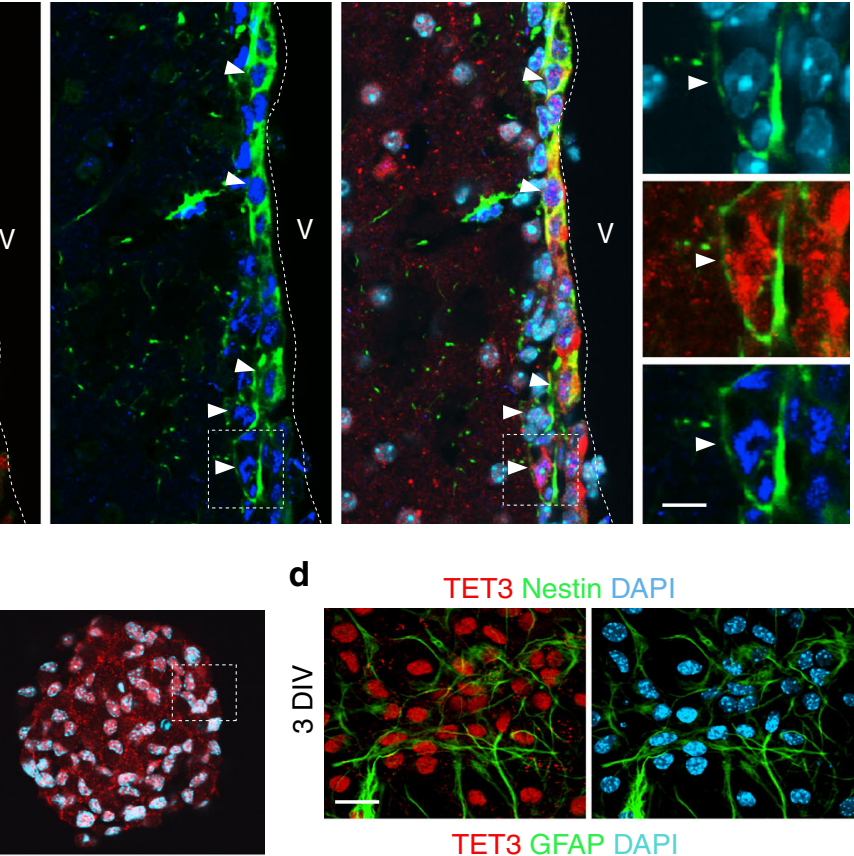

DAPI

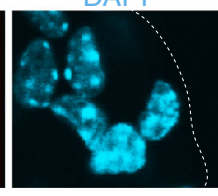

d

TET3 Nestin DAPI

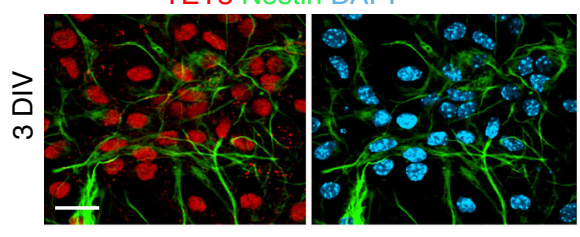

TET3 GFAP DAPI
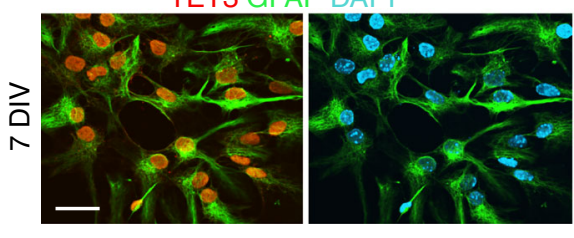

Fig. 1 TET3 is highly expressed in adult NSCs in vivo and in vitro. a qPCR for Tet1, Tet2 and Tet3 genes in different cell types and tissues. Tet1 is low expressed in adult NSCs and Tet2 downregulates postnatally. Tet 3 is highly expressed in the NSC population and maintained postnatally. Significant levels of Tet3 were also observed in NSCs after 2, 3 and 7 days (DIV) of differentiation in vitro. P: postnatal day. Embryonic stem cell lines (ESCs) were used as a control of expression. Data are expressed relative to Gapdh. Mean is indicated in the boxplots as "+". b Immunohistochemistry images for TET3 (red), GFAP (green), and SOX2 (blue) in the SVZ of adult wild-type mice. High-magnification images are shown. Dark arrowheads indicate triple positive astrocytes cells. $\vee$ lateral ventricle lumen. c Immunocytochemistry for TET3 (red) and SOX2 (green) in proliferating neurospheres isolated from the adult SVZ. $\mathbf{d}$ Immunocytochemistry for TET3 (red) and Nestin (green) or GFAP (green) in adult NSCs after 3 and 7 days of differentiation. DAPI was used to counterstain DNA. One-way ANOVA and Tukey post-test were applied. All error bars show s.e.m. Number of samples used is indicated. Scale bars, $20 \mu \mathrm{m}$ (high-magnification images, $7 \mu \mathrm{m}$ ). Source data are provided as a Source Data file

doublecortin $+(\mathrm{DCX}+)$ neuroblasts (Supplementary Figure 1a). TET3 staining was also observed in mature neurons of the striatal parenchyma (Supplementary Figure 1b). Furthermore, TET3 was present in all proliferating SOX $2+$ cells in neurosphere cultures from the adult SVZ (Fig. 1c). Consistent with the analyses at the mRNA level the TET3 protein was detected in Nestin+progenitors and GFAP+astrocytes at 3 and 7 days of in vitro differentiation, respectively (Fig. 1d). Taken together, these data suggested a role for TET3 in adult NSCs.
In order to evaluate the function of TET3 in the adult SVZ, a murine genetic model was generated by crossing male mice carrying loxP sites flanking part of the Tet3 gene $(\text { Tet } 3 \text { loxp/loxp })^{42,43}$ with female mice expressing the Cre-recombinase under the control of the Gfap promoter (Gfap-cre $\left.{ }^{+/ 0}\right)^{44}$. Importantly, X-gal histochemistry and immunostaining for $\beta$-galactosidase in the adult brain of ROSA26R;Gfap-cre ${ }^{+/ 0}$ mice (Gfap-cre/LACZ) and control mice (Gfap-control/LACZ) showed positive staining in the SVZ, RMS and olfactory bulbs of the adult Gfap-cre/LACZ brains 
a

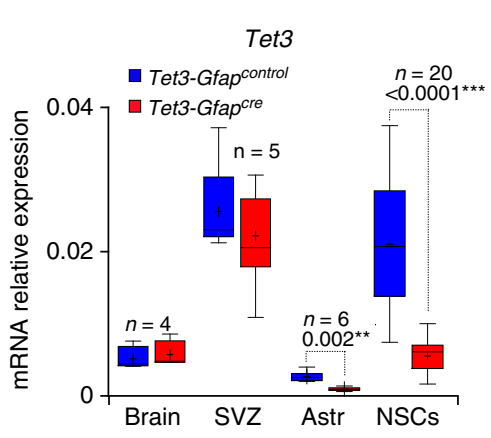

b

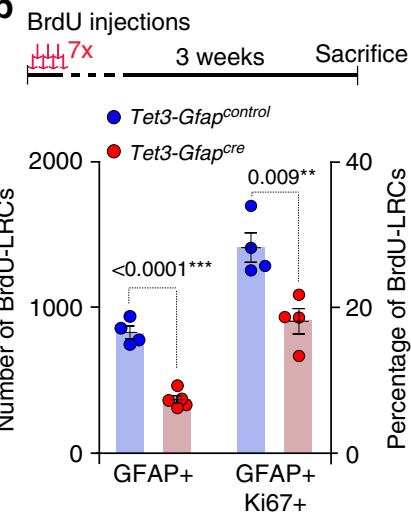

C

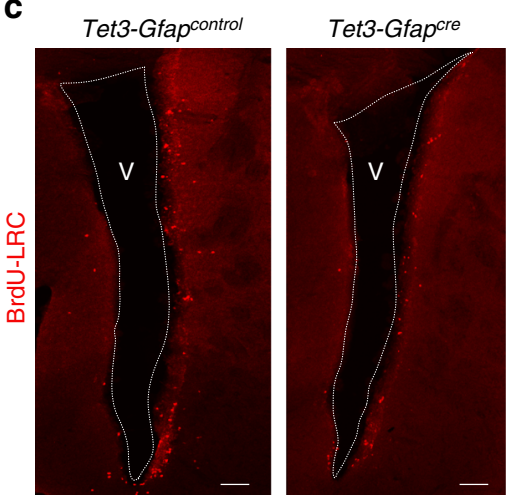

d

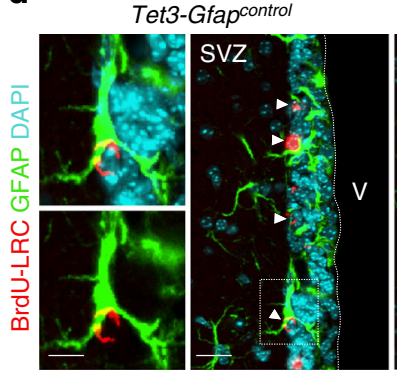

g

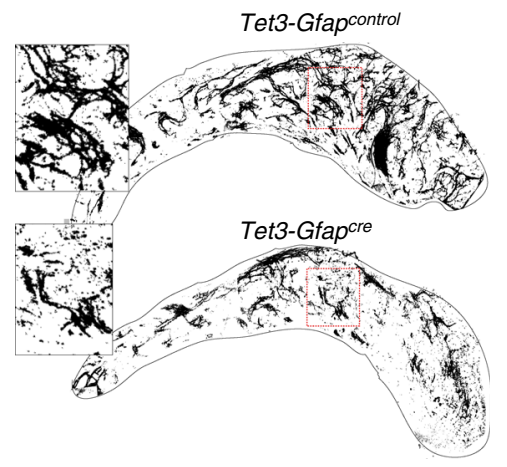

Tet3-Gfapcre

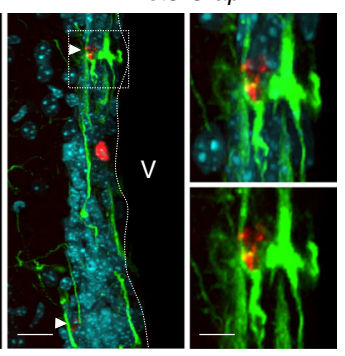

e

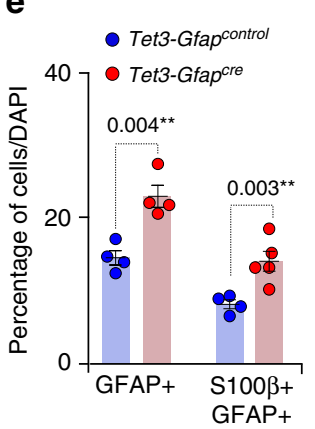

f
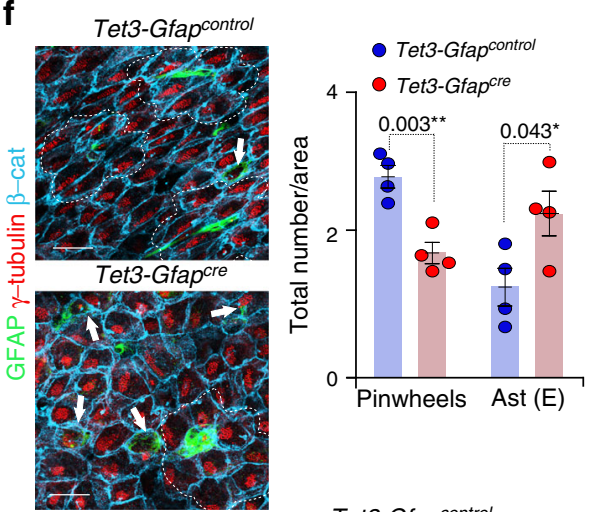

Percentage of DCX+ cells/DAPI

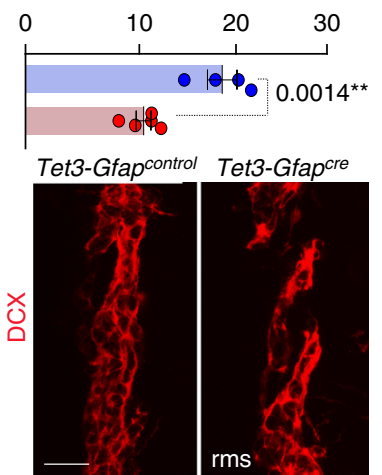

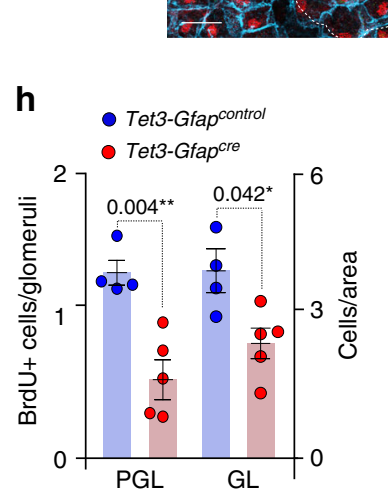

Tet3-Gfapcontrol

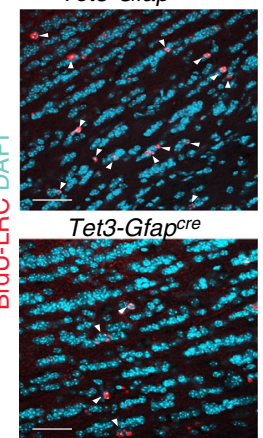

Fig. 2 Removal of Tet3 in the SVZ GFAP+population causes a depletion of the neural stem cell pool in vivo. a qPCR for Tet3 in adult brain, SVZ, astrocytes and NSCs from Tet3-Gfapcontrol and Tet3-Gfapcre mice. Data are expressed relative to Gapdh. Mean is indicated in the boxplots as "+". b Schematic drawing of the BrdU injection protocol (upper panel). Number of BrdU-label-retaining cells (BrdU-LRCs) that are GFAP+and GFAP/Ki67+in the SVZ of Tet3Gfapcontrol and Tet3-Gfapcre mice (lower panel). c Immunohistochemistry panoramic images of Tet3-Gfapcontrol and Tet3-Gfapcre SVZ stained for BrdU labelretaining cells (LRCs; red). d Immunohistochemistry images for BrdU-LRCs (red) and GFAP (green) in the SVZ of mice from both genotypes. Doublepositive cells are indicated with arrowheads. e Percentage of GFAP+and S100ß/GFAP+astrocytes cells in the SVZ of Tet3-Gfap control and Tet3-Gfap ${ }^{\text {cre }}$ mice. f Immunohistochemistry images for GFAP (green), $\gamma$-tubulin (red) and $\beta$-catenin (blue) in SVZ whole-mount preparations identifying pinwheel structures in both genotypes (left panels). Total number of pinwheels and differentiated astrocytes, found in the SVZ of Tet3-Gfapcontrol and Tet3-Gfapcre mice (right panels). $\mathbf{g}$ Confocal reconstructions of whole-mount staining for the neuroblast marker DCX in the rostral migratory stream (RMS) of mice from both genotypes. Inserts show high-magnification images of DCX+chains (left panels). Graph shows the quantification of the percentage of DCX+cells. Immunohistochemistry images for DCX (red) in the SVZ of Tet3-Gfapcontrol and Tet3-Gfap cre mice (right panels). $\mathbf{h}$ Quantification of the number of newborn neurons incorporating in the granular (GL) and periglomerular (PGL) layers in the olfactory bulbs (OB) of Tet3-Gfap control and Tet3-Gfap ${ }^{\text {cre }}$ mice (left panel). Immunohistochemistry images for BrdU-LRCs (red) in the granular layer of the OBs from both genotypes. $V$ lateral ventricle lumen. DAPI was used to counterstain DNA. All error bars show s.e.m. Unpaired two-tailed Student's $t$-test was used. $P$-values and number of mice used are indicated. Scale bars in (c), $100 \mu \mathrm{m}$; in (d and $\mathbf{g}$ ), $30 \mu \mathrm{m}$ (inserts in (d), $7 \mu \mathrm{m}$ ); in (f), $15 \mu \mathrm{m}$; in (h), $40 \mu \mathrm{m}$. Source data are provided as a Source Data file

(Supplementary Figure 2a) and colocalized with GFAP+cells (Supplementary Figures $2 \mathrm{~b}, \mathrm{c}$ ), corroborating the specific deletion of Tet3 in the adult GFAP+stem cell population. Significant expression of Cre-recombinase in Tet $3^{\text {loxp/loxp }-G f a p-c r e ~}{ }^{+10}$ (Tet3$G_{f a p^{c r e}}$ ) compared to Tet3 $3^{\text {loxp/loxp_Gfap-cre }}{ }^{0 / 0}$ (Tet3-Gfap ${ }^{\text {control }}$ ) control mice was confirmed in SVZ-derived NSCs (Supplementary
Figure 2d). Cre expression in NSCs isolated from Tet3-Gfap ${ }^{\text {cre }}$ deficient SVZ correlated with reductions in Tet3 mRNA and protein without compensatory changes in Tet1 and Tet2 (Fig. 2a and Supplementary Figures $2 \mathrm{e}-\mathrm{g}$ ). As expected, body and brain weights were not affected in Tet3-Gfap ${ }^{\text {cre }}$ mice compared to controls (Supplementary Figures 2h). 
Two-month-old Tet3-Gfap cre and Tet3-Gfap ${ }^{\text {control }}$ mice were injected with the nucleoside analog BrdU three weeks before sacrifice (Fig. 2b). BrdU is specifically retained in some slowly proliferating NSCs (label-retaining cells, LRCs) and in OB newborn neurons that ceased to divide and underwent terminal differentiation soon after the injection ${ }^{45}$. The number of GFAP +LRCs was significantly reduced in TET3-deficient mice (Fig. 2b$\mathrm{d}$ and Supplementary Figure $3 \mathrm{a}$ ) and fewer of them were positive for the proliferation antigen Ki67 (Fig. 2b and Supplementary Figure $3 \mathrm{~b}$ ), suggesting a role for TET3 in regulating the number of activated NSCs within the SVZ. Conversely, related to the decrease in activated NSCs in Tet3-deficient mice, we found a higher proportion of GFAP+cells with detectable levels of the calcium-binding protein $S 100 \beta$, a marker of terminally differentiated astrocytes (Fig. 2e). Type B cells are elongated and bear an apical process that extends through the cells of the ependymal monolayer, to end in a primary cilium immersed in the cerebrospinal fluid ${ }^{8}$. Monociliated B cells form structures known as pinwheels, and can, therefore, be readily identified in wholemount preparations of the SVZ, following immunofluorescence for GFAP and the basal body marker $\gamma$-tubulin. In line with the previous findings, we observed a decrease in the number of GFAP $/ \gamma$-tubulin + Type B cells contacting the ventricle in wholemounts of the Tet3-Gfap cre SVZ (Fig. 2f). Interestingly, we also found increased proportions of monociliated GFAP+cells with cell bodies completely intercalated within the ependymal layer, a feature that has been reported to increase with aging ${ }^{46}$ (Fig. 2f). Less densely populated DCX+neuroblast chains in the RMS (Fig. 2g) and fewer BrdU+newly-generated neurons in the granular and periglomerular layers of the $\mathrm{OB}$ were found in Tet3Gfap ${ }^{c r e}$ mice (Fig. 2h) likely as a result of the reduced number of NSCs in the TET3 mutant SVZ. However, no changes were observed in the density of newly-formed oligodendrocytes, scored as BrdU-LRC/OLIG2+cells in the corpus callosum (CC) of Tet3deficient mice (Supplementary Figure 3c). These data together indicate that TET3 is required for the maintenance of the NSC pool in the SVZ of adult mouse brains by preventing their premature differentiation into terminally differentiated astrocytes thus limiting neurogenesis in vivo.

Tet3 is required to maintain self-renewal of adult NSCs. Individual cells dissected from the postnatal SVZ can proliferate in medium containing basic fibroblast growth factor (FGF2) and/or epidermal growth factor (EGF) to produce multipotent clonal aggregates, called neurospheres ${ }^{45,47}$. To investigate cell-intrinsic properties of TET3, we derived neurospheres from adult

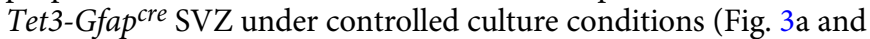
Supplementary Figure 4a). Expression of cre-recombinase and downregulation of Tet 3 was again confirmed in cultured neurospheres (Fig. 2a and Supplementary Figures 2d, e). SVZ isolated from Tet3-Gfap ${ }^{\text {cre }}$ adult mice yielded fewer primary neurospheres compared to those of controls (Fig. 3b). To assess specifically the self-renewal capability of these cultures, primary neurospheres were individually dissociated into single cells and plated at limiting dilution $(2.5$ cells $/ \mu \mathrm{l})$ for several passages (Fig. 3a). Tet3deficient neurospheres produced a significantly reduced number of new secondary clones at different passages (Fig. 3b, c). In contrast, TET3 did not appear to regulate proliferation, as we found no changes in diameter in primary or secondary Tet3deficient neurospheres (Fig. 3d). A cell cycle analysis of Tet3Gfap ${ }^{\text {control }}$ and Tet3-Gfap ${ }^{\text {cre }}$ neurosphere cultures revealed no changes in the proportion of cells in different cell cycle phases (Fig. 3e). The reduction in the number of neurospheres could not be ascribed to a decrease in cell survival as evidenced by similarly low proportions of caspase 3 +apoptotic cells in neurosphere cultures from the two genotypes (Fig. $3 \mathrm{e}$ and Supplementary Figure $4 \mathrm{~b}$ ). As a result of the self-renewal defect, the bulk growth expansion rate of Tet3-Gfap cre cultures after several passages was impaired compared to the Tet3-Gfap ${ }^{\text {control }}$ cells (Fig. 3f). These findings indicate that TET3 sustains self-renewal without affecting the overall proliferation rate or cell survival of adult NSCs.

TET3 prevents terminal astrocytic differentiation of NSCs. Adult NSCs are multipotent cells that can form neurons, astrocytes, and oligodendrocytes ex vivo. Indeed, when neurosphere cultures are induced to differentiate in vitro they undergo an orderly series of intermediate steps of proliferation and cell fate restriction resembling those occurring in vivo ${ }^{48,49}$ and resulting in the differentiation of derived neural progenitors into different neural cell types (Supplementary Figure 4a). Because the in vivo data suggested that Tet 3 deficiency resulted in increased astrocytic differentiation, we monitored the in vitro behavior of neural progenitors derived from Tet3-Gfap ${ }^{\text {cre }}$ and control neurospheres plated under adherent conditions in the absence of mitogens. During the first 2 days (2-DIV) of differentiation, a decrease in the neural progenitor marker Nestin together with an increase in the terminally differentiated astrocytic marker $S 100 \beta$ was observed in Tet3-Gfap ${ }^{\text {cre }}$ compared to control cultures (Fig. 3g), whereas no change in the levels of expression of the neuronal $\beta$ III-tubulin gene (Tubb3) was found (Supplementary Figure 4c). At the protein level, the deficiency in TET3 resulted in reduced proportions of Nestin+cells after 2-DIV of differentiation (Fig. 3h, i). Higher proportions of cells that were strongly positive for S100 $\beta$, a protein largely absent from neurogenic GFAP+cells correlating with loss of neurosphere-forming potential ${ }^{50}$, was also observed after 7-DIV of differentiation (Fig. 3h, i). Conversely, neuronal and oligodendroglial differentiation was normal as no significant differences were found in the percentage of $\beta$ IIItubulin+or O4+cells, respectively (Supplementary Figures $4 \mathrm{~d}, \mathrm{e}$ ).

In order to test whether enhanced astrocytic differentiation in the absence of Tet3 was consistently accompanied by a reduction in the capacity of NSCs to form neurospheres, 7-DIV differentiated NSCs cultures were detached and replated under proliferating conditions with mitogens (Supplementary Figure 4a). This led to the reactivation of a small proportion of cells with the capacity to form neurospheres in non-adherent conditions after 12 days in vitro (12 DIV) (Supplementary Figure 4f). The number of neurosphere-forming cells in Tet3-Gfap ${ }^{\text {cre }}$ NSCs was significantly reduced, indicating that the bias toward a more differentiated astrocytic phenotype in the absence of TET3 correlated with a reduction in stemness (Supplementary Figure 4f). This suggests that TET3 can directly promote the neurogenic potential of the multipotential stem cell-like astrocytes by preventing their premature differentiation.

TET3 regulates NSCs maintenance through repression of Snrpn. To understand the molecular mechanism underlying the differentiation defects observed in Tet3-deficient NSCs, a RNAseq was performed in control and Tet3-Gfap cre neurosphere cultures. RNA-seq analysis confirmed the downregulation of Tet3 mRNA levels in Tet3-Gfap cre compared to control cultures (LogFC in Tet3-Gfap ${ }^{\text {cre }}$ neurosphere cultures: -1.0074 with a $\left.\mathrm{FDR}=3.97 \times 10^{17}\right)$. Tet3 depletion resulted in 97 significantly downregulated and 96 upregulated genes (Fig. 4a; Supplementary Figure 5a; Supplementary Data set 1 and 2). Interestingly, Gene Set Enrichment Analysis (GSEA) revealed specific changes in pathways promoting cell growth such as PI3K-Akt, MAPK, and Wnt signaling pathways and genes involved in synaptic plasticity

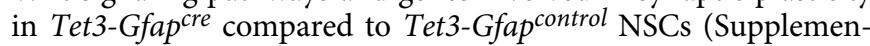
tary Figure 5b). 


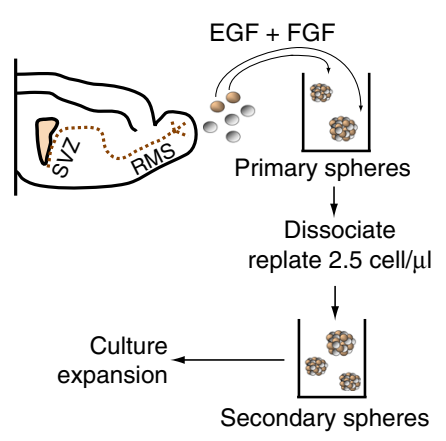

b

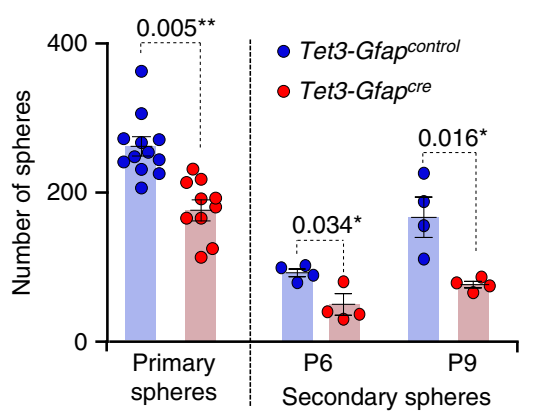

c

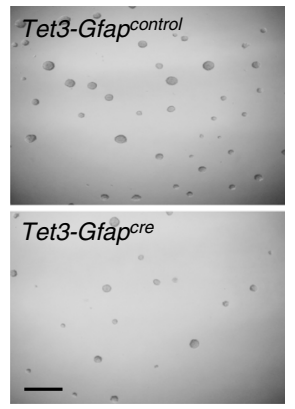

d

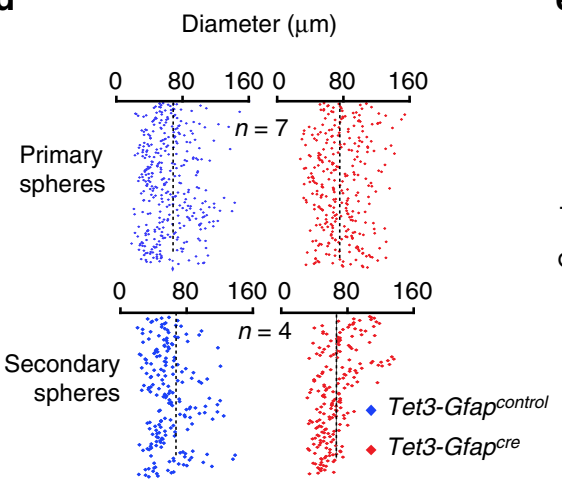

g

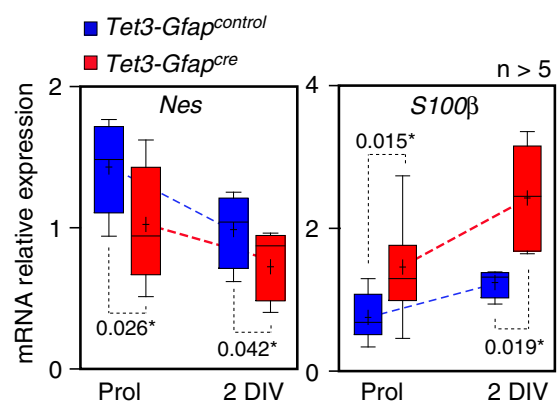

e

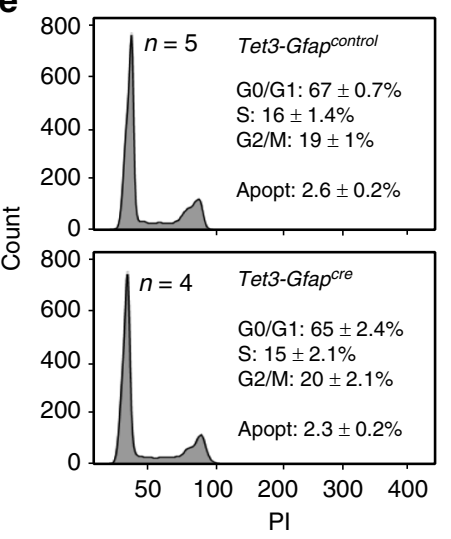

h

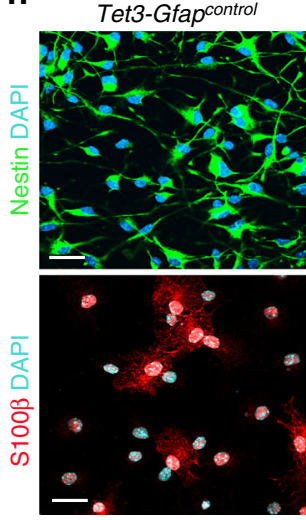

f

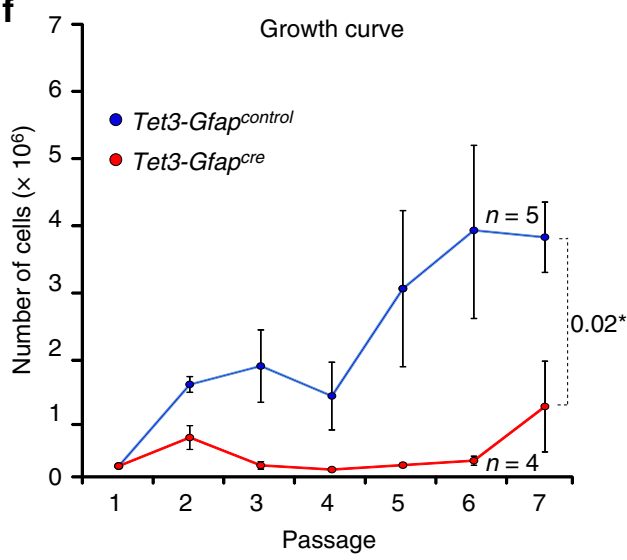

i
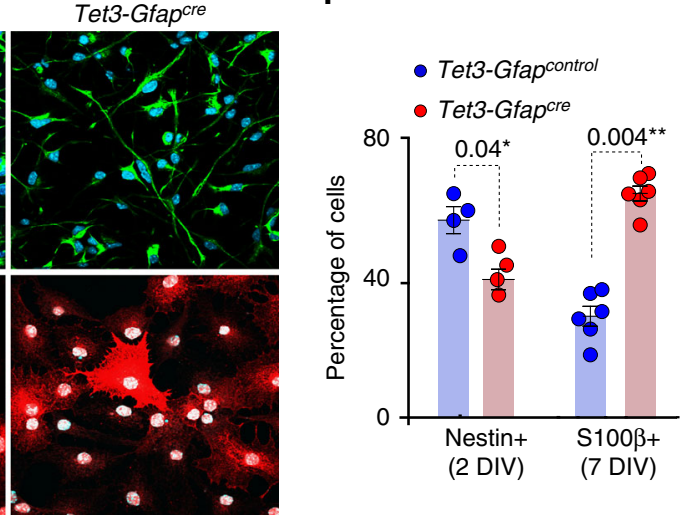

Fig. 3 Tet3 deficiency causes a decrease in NSCs self-renewal triggering their terminal differentiation into non-neurogenic astrocytes. a Schematic representing the primary and secondary forming neurosphere assay and the expansion protocol. Neurospheres are cultured in proliferation conditions with the mitogens EGF and FGF. Cells are dissociated and replated at 2.5 cells/ $\mu$. Secondary neurospheres are counted 5 days later. $\mathbf{b}$ Number of primary neurospheres obtained from the SVZ of Tet3-Gfapcontrol and Tet3-Gfap cre mice. Number of secondary spheres formed after passages (P) 6 and 9 is also shown. c Representative images of secondary neurosphere cultures from both genotypes. $\mathbf{d}$ Diameter of primary and secondary neurospheres formed in Tet3-Gfap ${ }^{\text {control }}$ and Tet3-Gfap cre cultures. Dashed lines indicate the mean diameter. e Cell cycle profiles in neurosphere cultures from both genotypes after 3 days in vitro. Percentages of G0/G1, S and G2/M phases are indicated. Percentages of apoptosis are also shown. $\mathbf{f}$ Growth curve showing the total number of cells formed after 7 passages in Tet3-Gfap control and Tet3-Gfap ${ }^{\text {cre }}$ neurosphere cultures. $\mathbf{g}$ qPCR for the undifferentiation marker Nestin and the terminally differentiated marker S100 $\beta$ in Tet3-Gfap control and Tet3-Gfapcre NSCs in proliferation conditions (Prol) and after 2 days of differentiation (2 DIV). Data are expressed relative to Gapdh. Mean is indicated in the boxplots as " + ". h Immunocytochemistry images for Nestin (green; upper panels) and S100 $\beta$ (red; lower panels) in NSCs of both genotypes after 2 or 7 days of differentiation, respectively. DAPI was used to counterstain DNA. i Percentage of cells

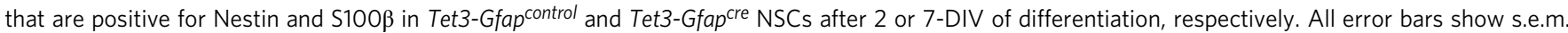
Unpaired two-tailed Student's t-test was used. P-values and number of samples are indicated. Scale bars in (c), $200 \mu \mathrm{m}$ and in (h), $40 \mu \mathrm{m}$. Source data are provided as a Source Data file

Non-canonical regulation of imprinted genes has been shown to regulate adult neurogenesis ${ }^{16,17,51}$. Moreover, the brain expresses high levels of $5 \mathrm{hmC}$, suggesting a potential contribution of TET3 to the epigenetic state at imprinted regions in adult NSCs. Based on the RNA-seq datasets, we next focused on changes in expression of all known imprinted genes (Supplementary Dataset 3). From around 150 imprinted genes analyzed, three showed a significant change in mRNA expression. Cntn3 (Contactin 3) was downregulated in Tet3-Gfap ${ }^{\text {cre }}$ NSCs, whereas Cobl (Cordon-bleu WH2 repeat) and Snrpn (Small nuclear 
a

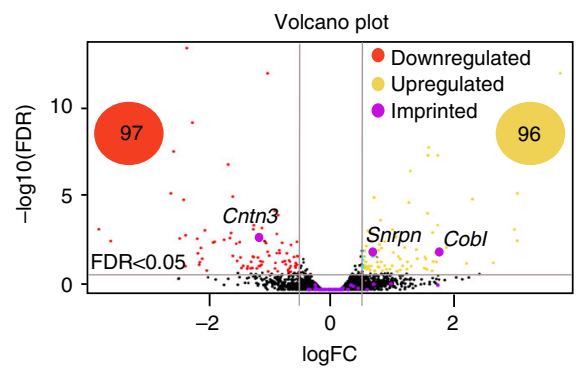

b

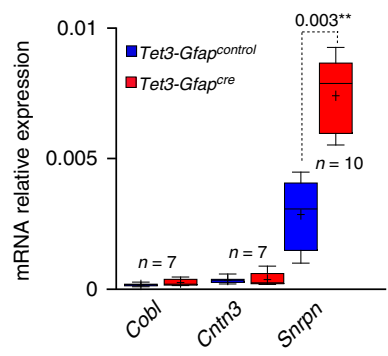

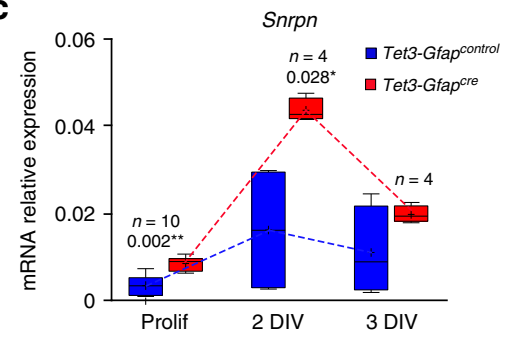

d

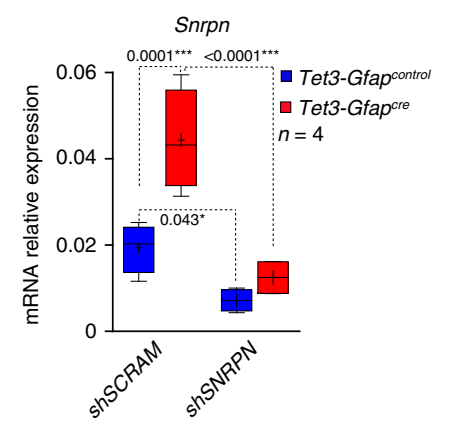

e

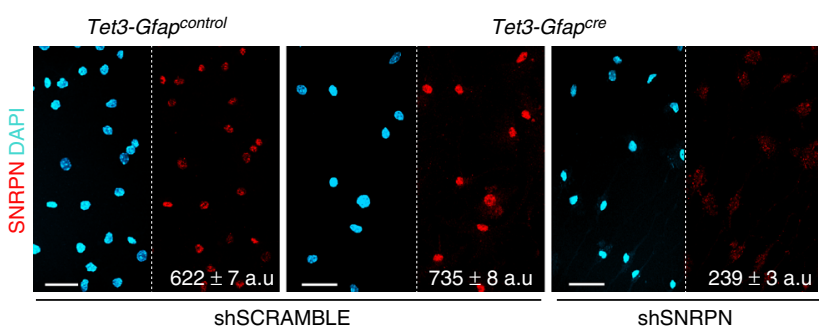

$\mathbf{f}$

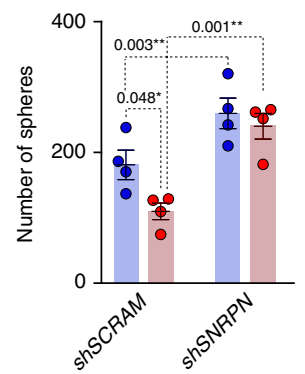

g

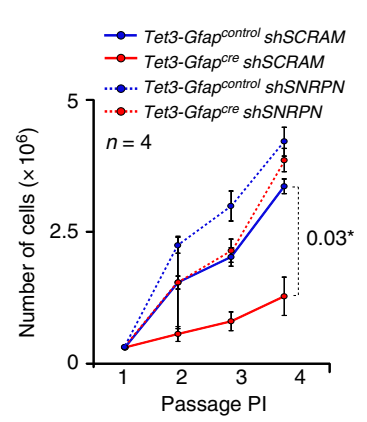

h

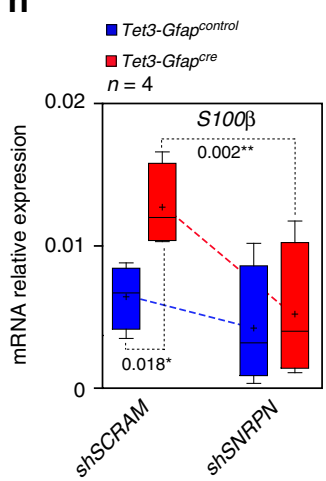

i

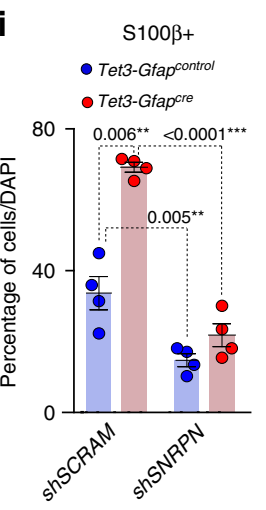

j

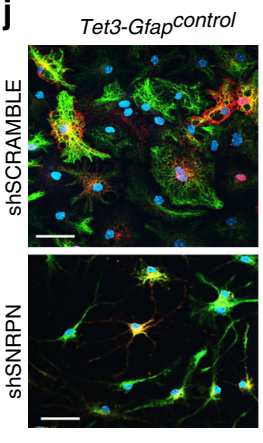

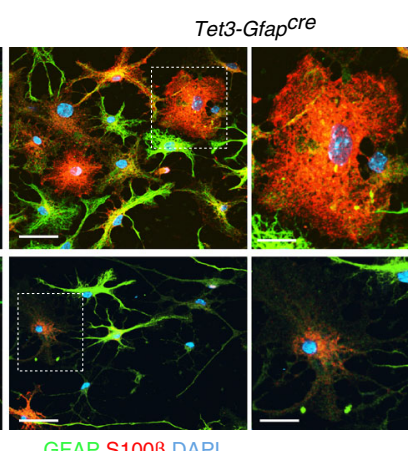

Fig. 4 Depletion of Tet3 in NSCs increases the expression of the imprinted gene Snrpn. a Volcano plot for genes differentially expressed by RNAseq in Tet3$\mathrm{Gfap}^{\mathrm{cre}}$ compared to control NSCs. Downregulated genes are in red and upregulated genes in yellow. Imprinting genes are showed in purple. $\mathbf{b}$ qPCR for Cntn3, Cobl, and Snrpn in NSCs from both genotypes. c qPCR for Snrpn in NSCs from both genotypes in proliferating conditions and after 2 or 3 days of differentiation. The pick of expression of the gene occurs after 2 DIV of differentiation. d qPCR for Snrpn in Tet3-Gfapcontrol and Tet3-Gfapcre NSCs that have been lentivirus transduced with a shRNA for SNRPN. A shRNA SCRAMBLE was used as a control. e Immunocytochemistry images for SNPRN (red) in Tet3Gfapcontrol and Tet3-Gfapcre NSCs 7 days after the shRNA experiment. f Number of secondary spheres formed after Tet3 downregulation in Tet3-Gfapcre cultures. $\mathbf{g}$ Growth curves showing the total number of cells formed after 4 passages in Tet3-Gfapcontrol and Tet3-Gfapcre neurosphere cultures that have been interfered with the shRNA for Snrpn. $\mathbf{h}$ qPCR for the astrocytic differentiation marker S100 $\beta$ after 2 DIV of differentiation in NSCs that have been lentivirus transduced with a shRNA for SNRPN. i Percentage of S100 $\beta+$ cells (relative to total number of DAPI) in shRNA interfered Tet3-Gfapcontrol and Tet3-Gfap ${ }^{\text {cre }}$ cultures. $\mathbf{j}$ Immunocytochemistry images for S100 $\beta$ (red) and GFAP (green) in interfered NSCs of both genotypes after 7-DIV of differentiation. Data are expressed relative to Gapdh. DAPI was used to counterstain DNA. All error bars show s.e.m. One-way ANOVA and Tukey post-test were applied. $P$-values and number of samples are indicated. Mean is indicated in the box and whiskers plots as " + ". Scale bars in (e and $\mathbf{j}), 40 \mu \mathrm{m}$ (high-magnification images $15 \mu \mathrm{m}$ )

ribonucleoprotein-associated polypeptide N) showed increased levels of expression in Tet3-deficient neurospheres compared to controls (Fig. 4a). Validation of the RNAseq data by qPCR showed very low levels of expression of Cntn3 and Cobl in NSCs but confirmed the significant upregulation of Snrpn in Tet3Gfap ${ }^{\text {cre }}$ neurospheres (Fig. 4b; Supplementary Figure 6a). A more detailed analysis of Snrpn expression in NSCs revealed that the gene was significantly expressed both in proliferating and differentiating conditions with a peak of expression at the first steps of the process of differentiation (Fig. 4c). Accordingly, although Snrpn was increased in Tet3-Gfap ${ }^{\text {cre }}$ compared to Tet3Gfap ${ }^{\text {control }}$ proliferating NSCs, the maximum difference of Snrpn
mRNA levels in both genotypes was observed after 2-DIV of differentiation (Fig. 4c).

In order to determine whether the augmented levels of Snrpn in Tet3-deficient NSCs might be functionally responsible for the loss of stemness and enhanced astrocytic differentiation of NSCs, a lentivirus (Lv)-mediated short hairpin (sh) RNA was used to silence Snrpn (shSNRPN) expression in Tet3-Gfap ${ }^{\text {cre }}$ neurosphere cultures. Downregulation of Snrpn was verified by qPCR and immunocytochemistry in transduced cells (Fig. 4d, e) and resulted in the reversion of the neurosphere-forming capacity of Tet3-deficient cells to wild-type levels (Fig. 4f and Supplementary Figure 6b). Accordingly, the growth expansion rate of 
NSCs was increased to normal in Tet3-Gfap cre neurosphere cultures infected with the shSNRPN (Fig. 4g). To assess whether this rescue in the self-renewal and expansion capacities in Tet3-deficient cultures might be due to an independent effect of Snrpn on proliferation, neurospheres size was determined, however no changes were found in mean diameter in any condition (Supplementary Figures $6 \mathrm{~b}, \mathrm{c}$ ) suggesting a functional role of Snrpn in NSCs self-renewal. Consistently, lentiviral delivery of the shSNRPN in Tet3-deficient cells restored S100 $\beta$ levels to normal after 2 and 7-DIV of differentiation (Fig. $4 \mathrm{~h}$ and Supplementary Figure 6d). Moreover, the percentage of S100 $\beta$ + cells in Tet3-Gfap ${ }^{\text {cre }}$ differentiated cultures was restored to wildtype levels (Fig. $4 \mathrm{i}, \mathrm{j}$ ). No changes in the levels of Tubb3 mRNA or in the proportion of $\beta$ III-tubulin+cells were observed after knockdown of Snrpn levels in Tet3-Gfap cre neurospheres (Supplementary Figures 6d,e). Notably, downregulation of Snrpn in Tet3-Gfap control cells, caused an increase in neurosphere formation and cell growth and decreased the proportion of differentiated S100 $\beta+$ cells (Fig. $4 \mathrm{~d}-\mathrm{j}$ ). These data suggested that TET3 directly promotes the neurogenic potential of multipotent stem cell-like astrocytes via regulation of Snrpn, antagonizing their premature terminal differentiation into mature astrocytes.

Snrpn controls BMP to mediate differentiation of NSCs. Because our data indicated that Tet3 deficiency results in increased astrogliogenesis, we focused on Bone Morphogenetic Proteins (BMPs), cytokines that are strong promoters of gliogenesis in vivo and in vitro ${ }^{10,52}$. Real-time PCR analysis of the expression of these molecules revealed an enrichment of Bmp2 mRNA in the absence of TET3 that was completely restored to normal levels by interference of the Snrpn gene (Fig. 5a). BMP ligands signal through a tetrameric complex that is formed by BMP type II receptor (BMPR-II) and different classes of BMP type I receptors. Activation of BMPR-IA or -IB results in the phosphorylation of SMAD1, SMAD5, and SMAD8 DNA binding proteins, which heterodimerize with co-SMAD4, and together translocate to the nucleus to regulate gene expression ${ }^{53}$. To test whether activation of the pathway in response to elevated levels of BMP2 was increased in Tet3-deficient NSCs, we used panspecific antibodies to pSMAD1 and pSMAD5 (pSMAD1/5). Treatment of wild-type NSCs with $20 \mathrm{ng} / \mathrm{ml} \mathrm{BMP2}$ resulted in increased immunofluorescent detection of nuclear PSMAD and premature differentiation of $S 100 \beta+$ astrocytes (Supplementary Figures $6 \mathrm{f}-\mathrm{h}$ ), indicating that differentiating NSCs are responsive to BMPs. Notably, increased levels of nuclear pSMAD1/5 were detected in Tet3-Gfap cre compared to Tet3-Gfapcontrol NSCs (Fig. 5b). Furthermore, this was antagonized by the addition of Noggin, a natural antagonist of BMP and by the downregulation of the Snrpn gene (Fig. 5b).

To further substantiate these data, we used a luciferase reporter construct that contains two copies of two distinct highly conserved BMP-responsive elements (BREs) and the associated regulatory motifs of the natural human and mouse Id1 promoter as a pSMAD activity sensor (BRE-tk-luciferase) ${ }^{54}$. Consistent with the data described above, we found an increase in the basal activity of the BRE reporter in Tet3-Gfap ${ }^{\text {cre }}$ when compared with wild-type cells, and this increase was efficiently abrogated by the addition of Noggin, as well as by the interference of Snrpn (Fig. 5c). Taken together, these results demonstrate that BMP signaling is augmented in the absence of Tet 3 and that control of BMP2 by SNRPN is functionally responsible for the enhanced astrocytic differentiation of Tet3-deficient NSCs.

A non-catalytic action of TET3 contributes to Snrpn repression. Significant levels of $5 \mathrm{mC}$ and $5 \mathrm{hmC}$ were found by immunocytochemistry in neurospheres isolated from the adult SVZ (Supplementary Figure 7a). Analysis of $5 \mathrm{hmC}$ and $5 \mathrm{mC}$ staining in vivo also showed variable intensities between different cellular populations within the adult SVZ (Supplementary Figures $7 \mathrm{~b}, \mathrm{c}$ ). In particular, distribution of $5 \mathrm{hmC}$ and $5 \mathrm{mC}$ showed high levels of $5 \mathrm{hmC}$ and low levels of $5 \mathrm{mC}$ in the GFAP/SOX2 +stem cell population (Supplementary Figures $7 \mathrm{~b}, \mathrm{c}$ ). In contrast, no $5 \mathrm{hmC}$ was found in the more differentiated cells such as the doublecortin (DCX)+neuroblast population (Supplementary Figure $7 \mathrm{~b}$ ). Thus, the presence of $5 \mathrm{hmC}$ in the NSC population along with its apparent exclusion from differentiated cells, a

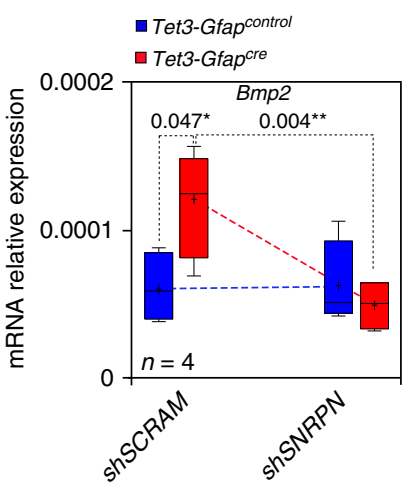

b

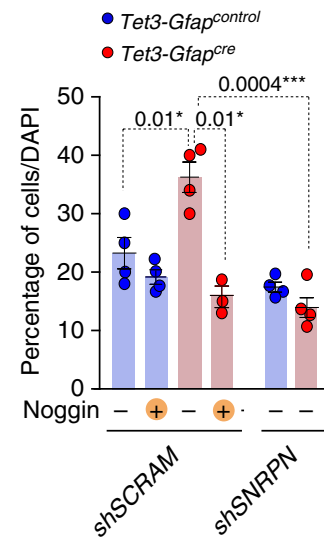

pSMAD1/5+ $0004^{* * *}$

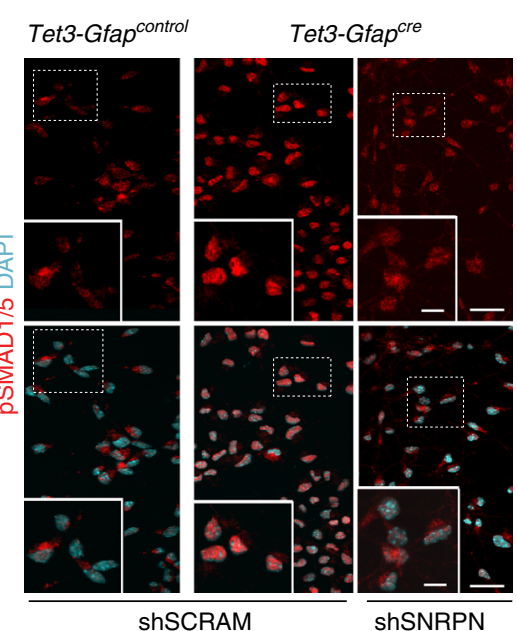

C

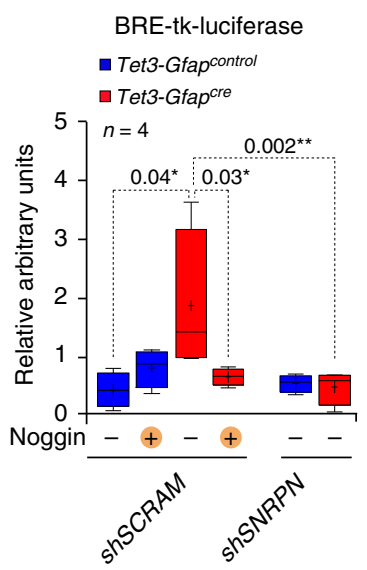

Fig. 5 BMP2 mediates terminal differentiation of Tet3-deficient NSCs. a qPCR for Bmp2 in Tet3-Gfapcontrol and Tet3-Gfapcre interfered NSCs after 2 DIV of differentiation. Data are expressed relative to Gapdh. b Percentage of pSMAD1/5+cells relative to total cells in Tet3-Gfapcontrol and Tet3-Gfapcre NSCs that have been interfered with a shRNA for SNRPN (left). NSCs were treated with $100 \mathrm{ng} / \mathrm{ml}$ of the BMP natural antagonist Noggin. Immunocytochemistry images for pSMAD1/5+cells (right). DAPI was used to counterstain DNA. c BRE-tk-luciferase reporter activity in Tet3-Gfap control and Tet3-Gfap cre shRNA interfered neurospheres and treated with Noggin. All error bars show s.e.m. One-way ANOVA and Tukey post-test were applied. P-values and number of samples are indicated. Mean is indicated in the box and whiskers plots as "+". Scale bars in (b), $40 \mu \mathrm{m}$ (high-magnification images $15 \mu \mathrm{m}$ ). Source data are provided as a Source Data file 
suggested that formation of $5 \mathrm{hmC}$ might also participate in the function and/or maintenance of the undifferentiated state in the adult neurogenic niches. Notably, upon Tet 3 knockdown no changes in global $5 \mathrm{mC}$ or $5 \mathrm{hmC}$ levels, determined by ELISA, were observed in adult Tet3-Gfap ${ }^{\text {control }}$ and Tet3-Gfap ${ }^{\text {cre }}$ neurosphere cultures (Supplementary Figure 7d). Consistently, immunohistochemistry for $5 \mathrm{hmC}$ within the Tet3-Gfap ${ }^{\text {cre }} \mathrm{SVZ}$ revealed no significant changes in global $5 \mathrm{hmC}$ in Tet3-deficient NSCs in vivo (Supplementary Figure 7c).

Snrpn belongs to the cluster of imprinted genes associated with Prader-Willi Syndrome and is canonically expressed from the paternally inherited chromosome ${ }^{55}$. The mouse Snrpn gene has a germline derived differentially methylated region (DMR) that is associated with imprinted Snrpn expression, and is required for imprinting control of the domain (Supplementary Figure 8a) ${ }^{56,57}$. We have previously shown the expected paternal expression of Snrpn in normal tissue and adult NSCs ${ }^{16}$, but loss of imprinting of the gene could explain the upregulation of Snrpn in Tet3$G f a p^{\text {cre }}$ NSCs. To test this, bisulfite sequencing of the Snrpn DMR was performed in Tet3-Gfap ${ }^{\text {control }}$ and Tet3-Gfap ${ }^{\text {cre }}$ neurosphere cultures (Supplementary Figure 8a, b). Both control and mutant neurospheres showed $50 \%$ methylation indicating retention of imprinting. Bisulfite sequencing cannot distinguish between $5 \mathrm{mC}$ and $5 \mathrm{hmC}$; therefore, to specifically assess $5 \mathrm{hmC}$ levels at the Snrpn DMR, a hydroxymethylation immunoprecipitation using antibodies against $5 \mathrm{hmC}$ (hydroxyMeDIP) was developed in Tet3-Gfap ${ }^{\text {control }}$ and Tet3-Gfap ${ }^{\text {cre }}$ NSCs cultures. Consistent with the bisulfite sequencing analysis, no differences in $5 \mathrm{hmC}$ levels were observed at the Snrpn promoter (Supplementary Figure 8c), suggesting that there was no loss of imprinting of the Snrpn gene.

The remarkable increase of Snrpn expression independently of a change to its DMR methylation and hydroxymethylation in Tet3-deficient NSCs, prompted us to explore the possibility that TET3 might have non-catalytic functions in NSCs. We first used chromatin immunoprecipitation followed by qPCR (ChIP-qPCR) to assess direct binding of TET3 to the Snrpn promoter in wildtype adult NSCs. Two Snrpn promoter regions (R1, -20 to +132 bp, and R2, +271 to $+434 \mathrm{bp}$ ) and Clcn 6 promoter, as a positive control region ${ }^{58}$, were chosen for analysis (Supplementary Figure 8a). Importantly, TET3 binding was observed at the Snrpn promoter regions but not at a more distal upstream region (-135 kb, Region 3, R3) (Fig. 6a). To specifically determine whether TET3 was bound to the paternal transcribed (unmethylated), maternal repressed (methylated) allele or to both alleles (Supplementary Figure 8a), a ChIP-qPCR for TET3 was performed in NSCs derived from adult F1 mice hybrids offspring from Mus musculus domesticus (C57BL6/J) females and Mus musculus castaneus (CAST/EiJ) males (BxC hybrids NSCs), in which two single-nucleotide polymorphisms (SNPs) were identified between the two subspecies at the Snrpn promoter (Supplementary Figure 8d). Direct sequencing of these SNPs after ChIP showed preferential binding for TET3 to the paternal transcribed and unmethylated allele (Fig. 6b), supporting a methylation-independent function of TET3 on the Snrpn gene.

To further determine whether TET3 contributes to transcriptional fine-tuning of Snrpn, we overexpressed TET3 in Tet3Gfap ${ }^{c r e}$ NSCs and found that this caused a significant decrease in Snrpn expression but not in other imprinted genes such as Mcts2 (Fig. 6c and Supplementary Figure 9a). Notably, when we overexpressed a catalytically inactive variant of TET3 (Tet3 CDmut) we observed a comparable decrease in the levels of the

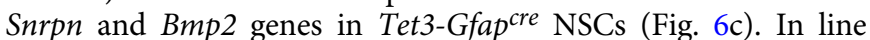
with this, overexpression of either wild type or catalytically inactive TET3 led to an increase in the number of spheres formed

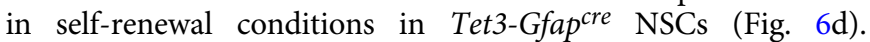
Consistently, overexpression of TET3 in Tet3-deficient cells restored the percentage of S100 $\beta+$ cells in Tet3-Gfap cre differentiated cultures to wild-type levels (Fig. 6e, f). Overexpression of TET3 in Tet3-Gfap control NSCs did not modify Snrpn and Bmp2 levels (Supplementary Figure 9b). Moreover, self-renewal and astrocytic differentiation were also normal in control cultures (Supplementary Figures 9c-e). However, when we overexpressed TET3 or its catalytically inactive variant in Tet3-Gfap ${ }^{\text {cre }}$ cultures, we observed a decrease in the levels of pSMAD1/5 and in the basal activity of the BRE reporter (Fig. $6 \mathrm{~g}, \mathrm{~h}$ ). These data together demonstrate that TET3 binds to the Snrpn promoter preferentially at the paternal allele to contribute to transcriptional repression of the gene in adult NSCs, independently of its catalytic function and that BMP2 signaling is responsible for the astrocytic terminal differentiation mediated by SNRPN (Supplementary Figure 10).

\section{Discussion}

The formation of new neurons in the adult brain throughout life involves the activation of pools of NSCs while preserving the stem cell number. Emerging evidence indicates that imprinted genes regulate the life-long function of adult NSCs ${ }^{16,17,51}$. This study demonstrates a role for the dioxygenase TET3 in the self-renewal and hence maintenance of NSCs within the adult SVZ. We show that loss of Tet3 results in the differentiation of neurogenic progenitors both in vivo and in vitro and compromises the maintenance of the NSC pool in this niche leading to reduced OB neurogenesis. More interestingly, our work indicates that TET3 regulates self-renewal by repressing transcription of the imprinted gene Snrpn through mechanisms that are independent of its catalytic function in modulating methylation.

TET3 deficiency causes an active apoptosis of neural progenitors derived from ESCs, which results in a reduction of neuronal production $^{30}$. This context differs from ours, as in adult NSCs Tet3 deficiency does not modify apoptosis or survival rates. Rather, when TET3 is absent in the GFAP+population in vivo, a depletion of the adult NSC pool is observed, and this is due to their terminal differentiation into non-neurogenic astrocytes. As a consequence of the continuous depletion of the NSC pool in Tet3-deficient mice, the process of neurogenesis to the $\mathrm{OB}$ is impaired, a finding recapitulated in neurosphere cultures in vitro. Interestingly, no changes in the proportion of the cell cycle phases are observed in mutant cultures indicating that TET3 promotes self-renewal capacity without affecting their overall proliferation rate. Differentiation of both mutant and wild-type NSCs into the three cell lineages of the central nervous system supports premature differentiation of NSC into non-neurogenic astrocytes in Tet3-deficient NSCs.

Gene expression analyses have revealed the molecular bases of these phenotypic observations, with a significant upregulation of the Snrpn gene in Tet3-deficient NSCs. Snrpn belongs to the Prader-Willi imprinted gene cluster and is expressed from the paternally inherited chromosome ${ }^{55}$. Patients with Prader-Willi syndrome lack expression of Snrpn and exhibit neurological problems including learning difficulties and hyperphagia ${ }^{59,60}$. Snrpn encodes the RNA-associated SmN (survival motor neurons) protein implicated in pre-mRNA editing, which contributes to tissuespecific alternative splicing ${ }^{61}$. It is well-established that Snrpn is highly expressed in brain and its expression increases markedly during postnatal brain development ${ }^{61}$. Our data indicate that TET3 regulates Snrpn dosage in an imprint-independent manner and that this is responsible for the premature differentiation of NSCs that we observe. Our findings suggest that Snrpn overexpression influences the NSC pool in adults, a function that has not previously been associated with Prader-Willi Syndrome and which has implications for our understanding of the etiology of this disorder. 
a

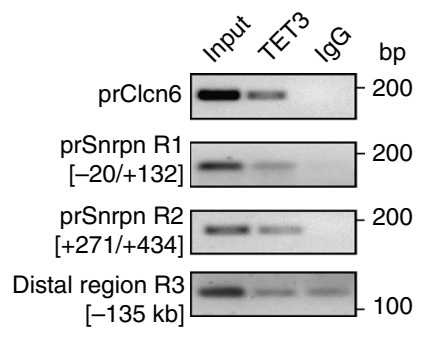

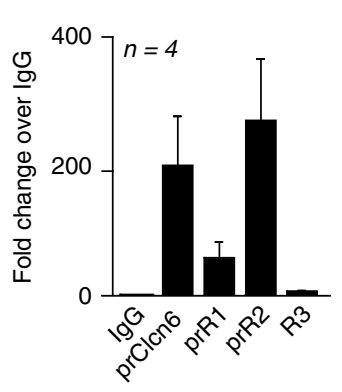

C

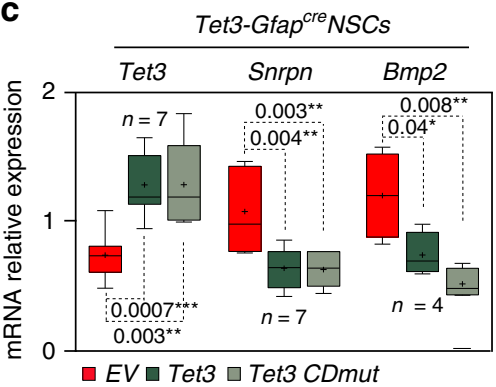

b

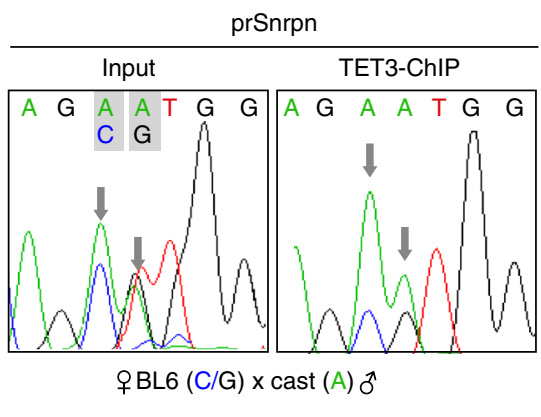

d

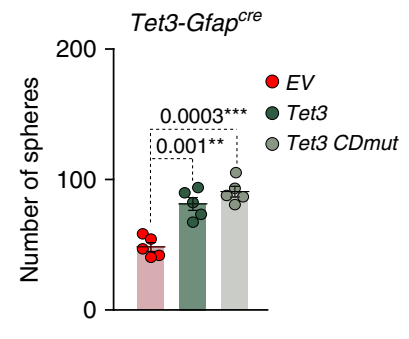

e
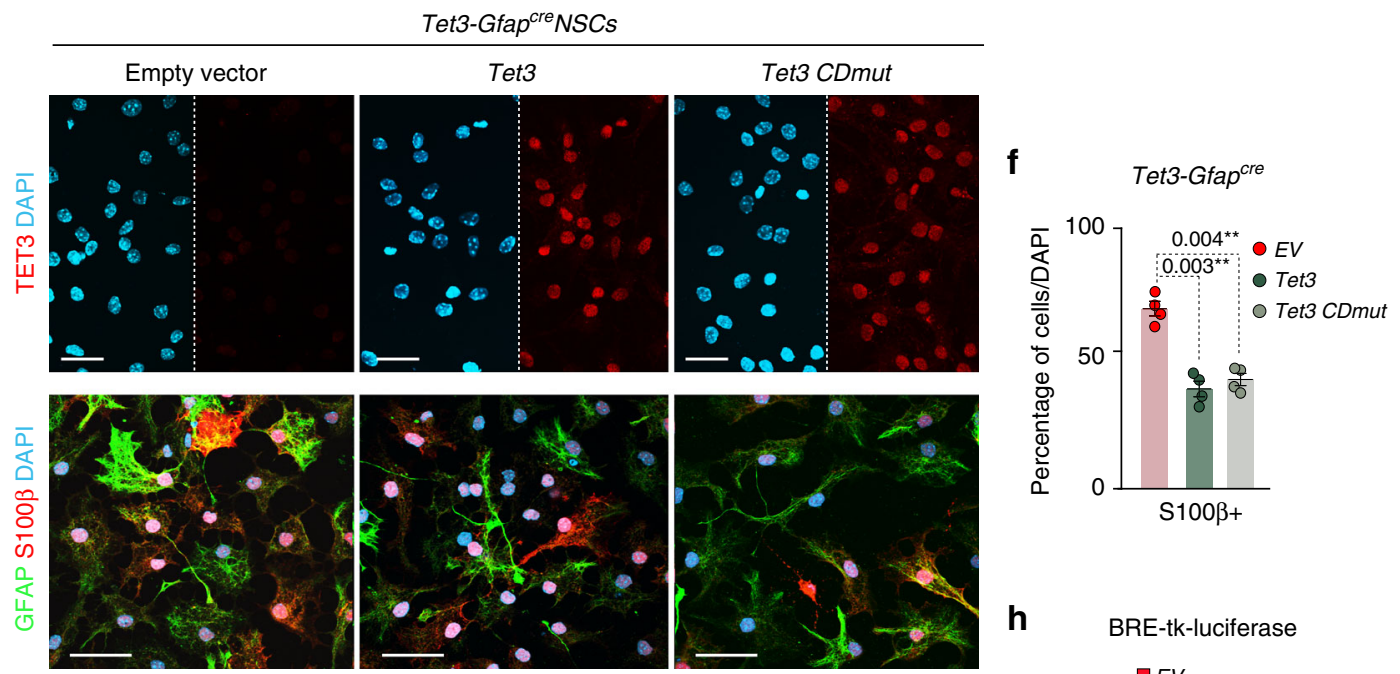

g
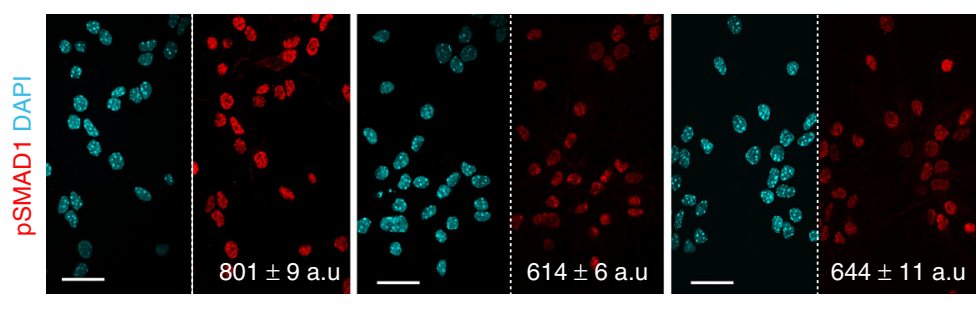

h

BRE-tk-luciferase

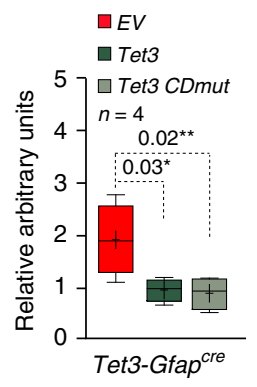

The effects of BMPs in astroglial differentiation have been reported extensively for both late fetal and postnatal progenitors $^{52,53}$, and most frequently, BMP-induced astroglial differentiation is related to deficiencies in the production of other cell lineages ${ }^{53}$. Moreover, BMP4 overexpression appears to accelerate the differentiation of some radial glia, suggesting that multipotential progenitors terminally differentiate when exposed to $\mathrm{BMPs}^{53,62}$. Our findings show that Snrpn overexpression correlates with elevated levels of this morphogen, suggesting that premature differentiation of Tet3-deficient NSCs into astrocytes may be partly

the consequence of the augmented activity of the BMP signaling. Moreover, the presence of the BMP antagonist Noggin that is produced by ependymal cells ${ }^{63}$ could be balancing the Snrpn function in the SVZ, preventing the differentiation of the NSC pool. Indeed, we showed that addition of Noggin to Tet3-deficient cultures, restored the terminal astrocytic differentiation phenotype observed. Further work will be necessary to elucidate whether Snrpn has a role on the pre-mRNA editing of BMP genes.

TET enzymes regulate the balance of DNA methylation and demethylation by dynamic conversion of $5 \mathrm{mC}$ into $5 \mathrm{hmC}, 5 \mathrm{fC}$, 
Fig. 6 TET3 binds to Snrpn promoter at the paternal allele to regulate its expression independently of methylation. a qPCR after chromatin immunoprecipitation (ChIP) of wild-type NSCs. Two regions (R1 and R2) of the Snrpn promoter (prSnrpn) were analyzed. Clcn6 promoter was used as a control for TET3 binding. A non-relevant distal region (R3) was analyzed as a control of specificity. Values are shown as the fold change over the IgG. b DNA sequences after ChIPqPCR for TET3 in F1 hybrid NSCs derived from Mus musculus domesticus (abbreviated, BL6) and Mus musculus castaneus (abbreviated, Cast) mice (BxC), showing the two diagnostic strain-specific polymorphisms at the Snrpn promoter in the input sample. A more abundant presence of the "A" nucleotide, corresponding to the paternal allele (Cast), is observed. c qPCR for Tet3, Snrpn and Bmp2 genes in Tet3-Gfapcre NSCs that had been nucleofected with Tet3 or with Tet3 with a mutated catalytic domain (Tet3 CDmut). An empty vector was used as a control for nucleofection. $\mathbf{d}$ Number of secondary spheres formed in Tet3-Gfap cre cultures after nucleofection with Tet3 variants. A rescue in the self-renewal capacity was observed. e Immunocytochemistry images for TET3 (red) in Tet3-Gfapcre NSCs that had been nucleofected with Tet3, Tet3 CDmut or EV (upper panels). Immunocytochemistry images for GFAP (green) and $\mathrm{S10O} \beta$ (red) are also shown (lower panels). f Percentage of S100 $\beta+$ cells in Tet3-Gfapcre NSCs that had been nucleofected with Tet3, Tet3 CDmut, or EV. g Immunocytochemistry images for pSMAD1/5+(red) in nucleofected Tet3-Gfap ${ }^{\text {cre }}$ NSCs. h BRE-tk-luciferase reporter activity in Tet3-Gfap ${ }^{\text {cre }}$ NSCs that had been nucleofected with Tet3, Tet3 CDmut, or EV. All error bars show s.e.m. One-way ANOVA and Tukey post-test were applied. P-values and number of samples are indicated. Mean is indicated in the box and whiskers plots as "+". Scale bars, $30 \mu \mathrm{m}$. Source data are provided as a Source Data file

and $5 \mathrm{CaC}^{40,41}$. Several other reports have shown non-catalytic functionalities of the TET proteins ${ }^{23,36}$. Concretely, TET3 has been implicated in the stabilization of thyroid hormones nuclear receptors promoting transcriptional activation where TET3 catalytic domain is not implicated and a TET3 catalytic dead mutant can rescue the developmental defects caused by TET3 knockdown ${ }^{64}$. Most notably, TET3 interacts with O-linked $\mathrm{N}$-acteylglucosamine transferases (OGT) to influence transcription ${ }^{34,35}$. Although our data show significant amounts of $5 \mathrm{hmC}$ in GFAPexpressing cells within the SVZ and in proliferating cells in vitro, global levels of $5 \mathrm{mC} / 5 \mathrm{hmC}$ are not altered in Tet3-deficient NSCs suggesting non-catalytic effects of TET3 in this neurogenic niche. Indeed, we demonstrate that TET3 binds to the Snrpn promoter at the paternal transcribed and unmethylated allele acting as a transcriptional repressor of the gene by a catalytic-independent activity mechanism, supporting a multifunctional role for TET3 in adult NSCs.

Adult niches preserve the undifferentiated property of their resident stem cells, but contribution of epigenetic mechanisms to this process has remained largely unexplored. Our data have elucidated the action of TET3, preventing terminal differentiation and exhaustion of NSCs by transcriptional repression of the Snrpn gene independently of its methylation. We also described how this regulation acts in concert with niche signals such as BMP and Noggin to modulate the proper differentiation of the NSC pool. Further work will be relevant to elucidate the structural, genetic and epigenetic requirements of this regulatory mechanism and its contribution to neurogenic phenotypes.

\begin{abstract}
Methods
Animals and in vivo manipulations. GFAP-cre (6.Cg- Tg(Gfap-cre) $73.12 \mathrm{Mvs} / \mathrm{J})$ mice were obtained from Jackson Laboratory and genotyped as described ${ }^{44}$. GFAP-cre mice were generated using a $15 \mathrm{~kb}$ mouse Gfap promoter cassette containing all introns, promoter regulatory elements, exons, and $2 \mathrm{~kb}$ of $3^{\prime}$ and $2.5 \mathrm{~kb}$ of $5^{\prime}$ flanking regions of the mouse Gfap gene ${ }^{44}$. Gfap expression is prevented by the removal of a small region in exon 1. Tet3 $3^{\text {loxplloxp }}$ mice contain LoxP sites flanking exon 5 of Tet 3 gene ${ }^{43}$. Expression of Cre-recombinase results in a deletion of this region and a frame-shift from exon 6 that affects all downstream exons until a premature stop codon in exon 7. To generate specific deletion of Tet 3 in GFAP positive cells, heterozygous GFAP-cre transgenic animals were bred to Tet3 ${ }^{\text {loxp}}$ /loxp. Additionally, The Jackson laboratory reports that the GFAP-cre line have cre expression in the male germline. To avoid this problem, GFAP-cre females and Tet $3^{\text {loxp/loxp }}$ males were used to generate the experimental animals. Animals were genotyped by PCR analysis of DNA, extracted from mouse ear-punch tissue with the following primers: for the presence of cre-recombinase $C r e-F$ and $C r e-R$ and for the presence of LoxP sites Tet3F, Tet3R1, and Tet3R2 (Supplementary Table 1). Mice were maintained on a C57BL6 background. We have complied with ethical regulations for animal testing and research and experiments were carried out following protocols approved by the ethics committee of the University of Valencia (Spain).
\end{abstract}

Immunohistochemistry and $\boldsymbol{\beta}$-galactosidase staining. BrdU administration regimes have been previously detailed ${ }^{16,45}$. Briefly: mice at $2-4$-months-of-age were injected intraperitoneally with $50 \mathrm{mg}$ of BrdU per kg of body weight every $2 \mathrm{~h}$ for
12 consecutive hours ( 7 injections in total). Three weeks after the injections, animals were deeply anaesthetized and transcardially perfused with $4 \%$ paraf ormaldehyde (PFA) in $0.1 \mathrm{M}$ phosphate buffer saline $\mathrm{pH} 7.4$ (PBS) and brains were vibratome-sectioned at $40 \mu \mathrm{m}$. For immunohistochemistry, sections were washed in PBS and blocked at room temperature for $1 \mathrm{~h}$ in PBS with $0.1 \%$ Triton X-100 supplemented with $10 \%$ Fetal bovine serum (FBS) and then incubated overnight at $4{ }^{\circ} \mathrm{C}$ with primary antibodies (Supplementary Table 2). For BrdU detection and $5 \mathrm{mC} / 5 \mathrm{hmC}$ immunostainings, sections were pre-incubated in $2 \mathrm{~N} \mathrm{HCl}$ for $20 \mathrm{~min}$ at $37^{\circ} \mathrm{C}$ and neutralized in $0.1 \mathrm{M}$ sodium borate ( $\mathrm{pH} 8.5$ ). Detections were performed with fluorescent secondary antibodies for $1 \mathrm{~h}$ at RT (Supplementary Table 3). For the study of pinwheel structures, we prepared SVZ whole-mounts using protocols established previously ${ }^{8}$. Briefly, the lateral walls of the lateral ventricles were dissected out and the resulting whole-mounts were fixed for $1.5 \mathrm{~h}$ in $4 \%$ PFA and washed overnight at $4{ }^{\circ} \mathrm{C}$ in $\mathrm{PB}$. Whole-mounts for pinwheel identification were washed three times in PBS containing $0.5 \%$ Triton X-100 for 15 min each, blocked for $2 \mathrm{~h}$ in $10 \%$ FBS and $2 \%$ Triton X-100 in PBS, then incubated for $48 \mathrm{~h}$ at $4{ }^{\circ} \mathrm{C}$ with primary antibodies for $\gamma$-tubulin to label basal bodies and $\beta$-catenin antibodies to delineate the cell membrane (Supplementary Table 2). After incubation with appropriate secondary antibodies (Supplementary Table 3), nuclei were counterstained with $1 \mu \mathrm{gml}-1$ of DAPI and the stained walls were mounted with Fluorsave (Calbiochem). Images were captured and analyzed with an Olympus FV10i confocal microscope (Olympus). For $\beta$-galactosidase

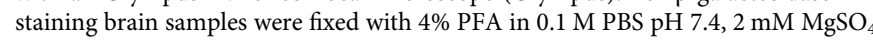
and $5 \mathrm{mM}$ EGTA for 30 minutes at $4{ }^{\circ} \mathrm{C}$ and processed for vibratome sectioning. Sections were incubated in PBS with $2 \mathrm{mM} \mathrm{MgCl}_{2}, 5 \mathrm{mM} \mathrm{K}_{3} \mathrm{Fe}(\mathrm{CN})_{6}, 5 \mathrm{mM} \mathrm{K}_{4} \mathrm{Fe}$ $(\mathrm{CN})_{6}, 0.01 \%$ sodium deoxycholate, and $0.02 \% \mathrm{NP}-40$ and $1 \mathrm{mg} \mathrm{ml}^{-1} \mathrm{X}-\mathrm{Gal}$ for $24 \mathrm{~h}$ at $37^{\circ} \mathrm{C}$ and washed several times in PBS.

Neurosphere cultures and knockdown experiments. Adult 2- to 4-months-old mice were killed by cervical dislocation. To initiate each independent culture, the brains of two different animals were dissected out and the regions containing the SVZ were isolated from each hemisphere and washed in Earle's balanced salt solution (EBSS; Gibco). Tissues were transferred to EBSS containing $1.0 \mathrm{mg} \mathrm{ml}^{-1}$ papain (Worthington DBA), $0.2 \mathrm{mg} \mathrm{ml}^{-1} \mathrm{~L}$-cystein (Sigma), $0.2 \mathrm{mg} \mathrm{ml}^{-1}$ EDTA (Sigma), and incubated for $20 \mathrm{~min}$ at $37^{\circ} \mathrm{C}$. Tissue was then rinsed in EBSS, transferred to Dulbecco's modified Eagle's medium (DMEM)/F12 medium (1:1 v/v; Life Technologies) and carefully triturated with a fire-polished Pasteur pipette to a single-cell suspension. Isolated cells were collected by centrifugation, resuspended in DMEM/F12 medium containing $2 \mathrm{mM}$ L-glutamine, $0.6 \%$ glucose, $9.6 \mathrm{~g} / \mathrm{ml}$ putrescine, $6.3 \mathrm{ng} / \mathrm{ml}$ progesterone, $5.2 \mathrm{ng} \mathrm{ml}^{-1}$ sodium selenite, $0.025 \mathrm{mg} / \mathrm{ml}$ insulin, $0.1 \mathrm{mg} \mathrm{ml}^{-1}$ transferrin, $2 \mu \mathrm{g} \mathrm{ml}^{-1}$ heparin (sodium salt, grade II; Sigma), and supplemented with $20 \mathrm{ng} \mathrm{ml}^{-1}$ epidermal growth factor (EGF; Invitrogen) and $10 \mathrm{ng} \mathrm{ml}^{-1}$ fibroblast growth factor (FGF; Sigma) ${ }^{45,48}$. For self-renewal assays, neurospheres were treated with Accutase $(0.5 \mathrm{mM}$; Sigma) for $10 \mathrm{~min}$, mechanically dissociated to a single-cell suspension and replated at low density $(2.5 \mathrm{cells} / \mu \mathrm{l})$ in growth medium (Fig. 3a). Neurospheres were allowed to develop for 6 days in a $95 \%$ air- $5 \% \mathrm{CO}_{2}$ humidified atmosphere at $37^{\circ} \mathrm{C}$. For culture expansion, cells were plated at a relatively high density $(10 \mathrm{cell} / \mu \mathrm{l})$ and maintained for several passages. For cell growth assessment, a fraction of the culture at any given passage point, consisting in 250,000 viable cells, was plated and the number of cells generated was determined at the time of the next passage. To generate the accumulated cell growth curves, the ratio of cell production at each subculturing step was multiplied by the number of cells at the previous point of the curve. This procedure was repeated for each passage. For bulk differentiation assays on secondary neurospheres, $80.000 \mathrm{cell} / \mathrm{cm}^{2}$ were seeded in Matrigel-coated coverslips and incubated 2 days in neurosphere culture medium without EGF. Medium was then changed with fresh medium without FGF but supplemented with $2 \%$ fetal bovine serum (FBS) for 5 more days (Supplementary Figure 4a). When indicated, neurosphere cultures were treated with BMP2 (R\&D Systems; $20 \mathrm{ng} / \mathrm{ml}$ ) or Noggin (R\&D Systems; $100 \mathrm{ng} / \mathrm{ml}$ ) at the time of plating. Cultures were fixed for staining at 2, 3, and 7 days of differentiation (DIV) with $4 \%$ PFA $0.1 \mathrm{M}$ PBS for $15 \mathrm{~min}$ and 
performed immunocytochemistry as described ${ }^{48}$. Primary and secondary antibodies and dilutions used are listed in Supplementary Table 2 and Supplementary Table 3, respectively. DAPI $(1 \mu \mathrm{g} / \mathrm{ml})$ was used to counterstain DNA. For cell cycle analysis $1 \times 10^{6}$ dissociated cells were stained using the BD Cycletest ${ }^{\mathrm{TM}}$ Plus DNA kit in accordance with the manufacture's protocol. Cell cycle phases were assessed in a FACS Verse flow cytometer (BD) and analyzed with FlowJo ${ }^{\circledR}$ software. Lentivirus containing a Snrpn-specific shRNA (TRC Clone number 0000109285, Sigma) or a non-target control (Mission SHC002, Sigma) plasmids were generated in HEK293T packing cells. Neurospheres freshly disaggregated were transduced with lentiviral particles for $6 \mathrm{~h}$. Lentivirus supernatant was removed and cells allow growing for experiments.

Luciferase reporter assays. For luciferase assays, we electroporated 2-2.5 $\times 10^{6}$ freshly disaggregated Tet3-Gfap ${ }^{\text {control }}$ and Tet3-Gfap ${ }^{\text {cre }}$ NSCs using the Amaxa NSC Nucleofector Kit, following the instructions provided by the manufacturer, with $2 \mu \mathrm{g}$ of the BRE-tk reporter construct driving the expression of the firefly luciferase and Renilla luciferase plasmid in a 1:20 ratio. We lysed cells after $48 \mathrm{~h}$ and obtained cell extracts using the Dual Luciferase Reporter kit (Promega), measured luciferase activity using a VICTOR3 reader and calculated the ratio of firefly to Renilla luciferase.

Expression studies. RNAs were extracted with RNAeasy mini kit (Qiagen) including DNase treatment, following the manufacturer's guidelines. For quantitative PCR, $1 \mu \mathrm{g}$ of total RNA was reverse transcribed using random primers and RevertAid H Minus First Strand cDNA Synthesis kit (Thermo Scientific), following standard procedures. Thermocycling was performed in a final volume of $10 \mu \mathrm{l}$, containing $1 \mu \mathrm{l}$ of cDNA sample and the reverse transcribed RNA was amplified by PCR with appropriate Taqman probes (Supplementary Table 4). Quantitative PCR was used to measure gene expression levels normalized to Gapdh, the expression of which did not differ between the groups. qPCR reactions were performed in a Step One Plus cycler with Taqman Fast Advanced Master Mix (Applied Biosystems). SYBR green thermocycling was also performed in a final volume of $12 \mu \mathrm{l}$, containing $1 \mu \mathrm{l}$ of cDNA sample, $0.2 \mu \mathrm{M}$ of each primer (Supplementary Table 1), and SYBR $^{\oplus}$ Premix ExTaq ${ }^{\text {TM }}$ (Takara) according to the manufacture instructions. A standard curve made up of doubling dilutions of pooled cDNA from the samples being assessed was run on each plate, and quantification was performed relative to the standard curve.

RNAseq. Library preparation and high-throughput sequencing were performed by the Central Service for Experimental Research (SCSIE) at the University of Valencia. RNA-seq libraries were generated from triplicated samples per condition using the Illumina TruSeq stranded mRNA Sample Preparation Kit v2 following the manufacturer's protocol. The RNA-seq libraries were sequenced using Illumina NextSeq 500. Analysis of the RNAseq data was performed by EpiDisease S.L. The sequences were aligned to the mouse genome reference (GRCm38.p5) taken from Ensembl using the Subread package in Linux. The expression of every gene per sample was measured using a gtf annotation file (GRCm38.87) taken from Ensembl and the R package Rsubread. Data have been deposited in Gene Expression Omnibus (GEO) with the series entry is GSE127815 (https://www.ncbi.nlm.nih. gov/geo/query/acc.cgi?acc $=$ GSE127815).

DNA methylation and pyrosequencing. DNA methylation level was quantified using bisulfite conversion and pyrosequencing. The DNA was bisulfited-converted using EZ DNA Methylation-Gold ${ }^{\mathrm{TM}}$ kit (Zymo research) in accordance with the manufacture's protocol. Specifically for Snrpn, bisulfite-converted DNA was amplified by PCR with specific primer pairs: Snrpn-DMR-F and Snrpn-DMR-R (Supplementary Table 1). PCRs were carried out in $20 \mu$ l, with $2 \mathrm{U}$ HotStar Taq polymerase (Qiagen), PCR Buffer $10 \times($ Quiagen), $0.2 \mathrm{mM}$ dNTPs, and $400 \mathrm{mM}$ primers. PCR conditions were: $96^{\circ} \mathrm{C}$ for $5 \mathrm{~min}$, followed by 39 cycles of $94^{\circ} \mathrm{C}$ for $30 \mathrm{~s}, 54{ }^{\circ} \mathrm{C}$ for $30 \mathrm{~s}$, and $72{ }^{\circ} \mathrm{C}$ for $1 \mathrm{~min}$. For pyrosequencing analysis, a biotinlabeled primer was used to purify the final PCR product using sepharose beads. The PCR product was bound to Streptavidin Sepharose High Performance (GE Healthcare), purified, washed with $70 \%$ ethanol, denatured with $0.2 \mathrm{~N} \mathrm{NaOH}$ and washed again with $10 \mathrm{mM}$ Tris-acetate. Pyrosequencing primer $(400 \mathrm{mM})$ was then annealed to the purified single-stranded PCR product and pyrosequencing was performed using the PyroMark Q96MD pyrosequencing system (Qiagen) using PyroMark $^{\circledR}$ reactives (Qiagen).

Immunoblotting and ELISA. Cells were lysed in cold RIPA buffer. Total protein concentration was determined using the BCA system (Pierce). Equal amounts $(30 \mathrm{mg})$ of protein were loaded on polyacrylamide gels for SDS-polyacrylamide gel electrophoresis. Proteins were transferred to polyvinylidene difluoride (PVDF) membranes and immunoblots were carried out using primary antibodies (Supplementary Table 2) followed by incubation with appropriate secondary horseradish peroxidase-conjugated antibodies (Supplementary Table 3) and chemoluminiscent detection (Western Lightning, PerkinElmer). All antibodies were diluted in PBS containing 5\% semi-skimmed milk and 0.1\% Tween-20. Proteins were revealed using Lightning ${ }^{\circledast}$ Plus ECL (Perkin Elmer) and the bands were analyzed by densitometry using Image (NIH) software. Uncropped scan of the WB gel is shown in Supplementary Figure 11a. For global determinations of $5 \mathrm{mC}$ and $5 \mathrm{hmC}$ contents, enzyme-linked immunosorbent assay (ELISA) was used. Double strand DNA was denatured at $98^{\circ} \mathrm{C}$ during $10 \mathrm{~min}$. DNA solutions were immobilized onto a 96-well plastic plate with Reacti-Bind DNA Coating Solution (Thermo Scientific) and incubated overnight at room temperature. Sample solutions were removed and plates were washed three times with washing buffer $(0.1 \mathrm{M}$ PBS containing $0.1 \%$ Tween-20) and blocked with blocking buffer (0.1 M PBS containing 5\% skimmed milk and $0.1 \%$ Tween-20) at room temperature for $1 \mathrm{~h}$. After washing three times with the washing buffer, primary antibodies for $5 \mathrm{hmC}$ or $5 \mathrm{mC}$ (Supplementary Table 2) were added and incubated at room temperature for $2 \mathrm{~h}$. After three more washes, secondary antibodies (HRP-conjugated goat antirabbit IgG for $5 \mathrm{hmC}$ or anti-mouse for $5 \mathrm{mC}, 1: 2000$ each, DAKO) were added and incubated at room temperature for $1 \mathrm{~h}$. After three washes, One-Step Ultra TMBELISA (Thermo Scientific) was added to the plate to develop chemiluminescent signals, and incubated at room temperature for $30 \mathrm{~min}$ or until the desired color appeared. Immediately, $2 \mathrm{M}$ sulfuric acid solution was added to the plate and the absorbance was measured at $450 \mathrm{~nm}$ using the 1420 Multilabel Counter VICTOR (Perkin Elmer). The amounts of hydroxymethylated and methylated DNA were obtained by comparison to a standard curve of hydroxymethylated and methylated DNA standards (5-hmC, 5-mC, \& cytosine DNA standard pack; Diagenode), respectively. The amount of total DNA examined was previously measured by the Quant-iT PicoGreen dsDNA Reagent and Kits (Invitrogen) according to the manufacturer's protocol. The percentage of $5 \mathrm{hmC}$ and $5 \mathrm{mC}$ was calculated by dividing the amount of hydroxymethylated and methylated DNA by that of total DNA, respectively.

ChIP and 5hmeDIP. For ChIP five $100 \mathrm{~cm}$ dishes with wild-type NSCs isolated from the adult SVZ were cross-linked and chromatin isolated. Chromatin was sheared to an average size of $200-500 \mathrm{bp}$ using a Bioruptor sonicator (Diagenode, UCD-200). Chromatin was pre-cleared with $10 \mu \mathrm{g}$ of non-immune rabbit IgG (Santa Cruz, cat. no. sc-2027) and $20 \mu \mathrm{l}$ of protein $\mathrm{G}$ magnetic beads (Dynabeads ${ }^{\circledR}$ cat. no. 10003D) for $3 \mathrm{~h}$ at $4{ }^{\circ} \mathrm{C}$ with rotation. A volume of $10 \mu \mathrm{g}$ of TET3 antibody (Supplementary Table 2) or rabbit IgG were added and incubated overnight at $4{ }^{\circ} \mathrm{C}$ on a rotation wheel. Chromatin was precipitated with $10 \mu \mathrm{l}$ protein $\mathrm{G}$ beads for $3 \mathrm{~h}$ at $4{ }^{\circ} \mathrm{C}$. An alicuot of chromatin before the immunoprecipitation was used a input. Beads were then washed followed by crosslink reversal and protein digestion. Finally, DNA was purified using MiniElute PCR purification kit (Qiagen) following manufacturer's instructions. To analyze the TET3 interaction with the Snrpn promoter, ChIP enriched DNA was analyzed by qPCR using SYBR green primers (Supplementary Table 1). Pull-downs using non-immune rabbit IgG were used as control for non-specific enrichments. The comparative Ct method was used to calculate fold enrichment levels normalizing to input DNA and non-specific IgG. Uncropped scans of the PCR electrophoresis are shown in Supplementary Figure 11b.

To study TET3 binding to the paternal or maternal alleles a ChIP for TET3 was performed in NSCs derived from adult F1 mice hybrids offspring from Mus musculus domesticus (C57BL6/J) females and Mus musculus castaneus (CAST/Ei]) males (BxC hybrids NSCs), in which we identified two SNPs between the two subspecies at the Snrpn promoter (Supplementary Figure 8d). SNP1 was a "C" nucleotide in BL6 and an "A" nucleotide in Cast mice. SNP2 was a "G" nucleotide in BL6 and an "A" nucleotide in Cast mice. Genomic DNA sequences were obtained after PCR with primers within Region 2 at the Snrpn DMR (Supplementary Table 1).

To enrich DNA samples in $5 \mathrm{hmC}$ modification, an immunoprecipitation for $5 \mathrm{hmC}$ was done using $5 \mu \mathrm{g}$ of sonicated DNA at 150-200 bp. DNA was denatured at $98{ }^{\circ} \mathrm{C}$ for $10 \mathrm{~min}$ and immediately cooled on ice for $10 \mathrm{~min}$. Milk buffer $(5 \%$ skimmed milk powder, $2 \mathrm{M} \mathrm{NaCl})$ and $10 x-I P$ buffer $(100 \mathrm{mM} \mathrm{Na}$-Phosphate $\mathrm{pH}$ 7.0, $0.5 \%$ TritonX-100) were added. DNAs were incubated with $0.5 \mu \mathrm{lof} 5 \mathrm{hmC}$ primary antibody (Supplementary Table 2) for $2 \mathrm{~h}$. In parallel, specific DNAbeads ${ }^{\oplus}$ Protein-G (Thermo Fisher) were washed with $0.1 \mathrm{M}$ PBS containing $1 \mathrm{mg} \mathrm{ml}^{-1}$ BSA for $2 \mathrm{~h}$. Beads were then collected with a magnet, added to the DNAs and incubated overnight at $4{ }^{\circ} \mathrm{C}$ with rotation. The day after, beads were collected using a magnet and the supernatant was kept (Unbound fraction, UB). Beads were washed several times with $1 \mathrm{x}$ IP buffer (10 mM Na-Phosphate $\mathrm{pH} 7.0,0.05 \%$ TritonX-100, $1 \mathrm{M} \mathrm{NaCl}$ ) and both fractions were treated with $35 \mu \mathrm{g}$ of Proteinase $\mathrm{K}$ (Roche) at $55^{\circ} \mathrm{C}$ for $30 \mathrm{~min}$. Finally, samples were purified using MiniElute PCR purification kit (Qiagen) following the manufacturer's instructions. To calculate $5 \mathrm{hmC}$ enrichment at the Snrpn-DMR, qPCR was done with specific primers (Supplementary Table 1). A standard curve made up of doubling dilutions of pooled aliquots from unbound fractions was run on each plate. Fold enrichment between bound and unbound fractions was calculated estimating relative concentration with the standard curve. To ensure the specificity of $5 \mathrm{mC}$ and $5 \mathrm{hmC}$ antibodies, genomic DNAs were spiked with $0.3 \mathrm{ng}$ of synthetic Arabidopsis sp. (Diagenode) sequence containing either $5-\mathrm{C}$ or $5 \mathrm{hmC}$ and subjected to immunoprecipitation. No cross-binding was detected (Supplementary Figure 8c).

Cloning of Tet3 variants and NSCs line construction. Tet 3 was cloned from cDNA from embryoid bodies and recombined into pDONR221 (Invitrogen). Mutations abolishing catalytic activity (HKD catalytic triad to YKA ${ }^{24,25}$ were introduced using the QuikChange II mutagenesis system (Agilent) using primers 
Tet3-mut-F (5'-GGACTTCTGTGCCCACGCCCACAAGGACCAACATAACCT CTACAATG-3') and Tet3-mut-R (5'-CATTGTAGAGGTTATGTTGGTCCTT GTGGGCGTGGGCACAGAAGTCC-3'). Variants were cloned into PB-DST-BSD (kind gift from Jose Silva) via Gateway cloning (Invitrogen) and are expressed constitutively from a CAG promoter. Adult NSCs lines harboring Tet 3 variants were generated by nucleofection of $2-2.5 \times 10^{6}$ freshly disaggregated NSCs with 3 $\mu \mathrm{g}$ of pIG-Tet 3 or pIG-Tet3CDmut plasmids using the Amaxa NSC Nucleofector Kit (Lonza, following the instructions provided by the manufacturer), plasmid integration via the piggyBAC system and selection with blasticidin $(10 \mu \mathrm{g} / \mathrm{ml})$. Control NSCs lines carrying a shSCRAMBLE were equally selected.

Statistical analysis. All statistical tests were performed using the SPSS software, version 24.0.0 for windows, and licended by the University of Valencia. The significance of the differences between groups was evaluated in all using the unpaired two-tailed Student's $t$-test or one-way ANOVA with Tukey post-hoc test when appropriate. Treatment experiments were analyzed by paired $t$-test. When comparisons were performed with relative values (normalized values and percentages), data were normalized by using an arc-sen transformation. Values of $P<0.05$ were considered statistically significant. Data are presented as the mean \pm standard error of the mean (s.e.m.) and the number of experiments performed with independent cultures or animals (n) and $P$-values are indicated in the figures. Box and whisker plots show the mean $(+)$, median (horizontal line in box), and maximum and minimum values.

Reporting summary. Further information on experimental design is available in the Nature Research Reporting Summary linked to this article.

\section{Data availability}

The data that support the findings of this study are available from the corresponding author upon reasonable request. Source data for figures 1a; $2 \mathrm{a}, \mathrm{b}, \mathrm{e}, \mathrm{f}, \mathrm{g}, \mathrm{h} ; 3 \mathrm{~b}, \mathrm{~d}, \mathrm{e}, \mathrm{f}, \mathrm{g}, \mathrm{I}$; 4a-d, 4f-i, 5a-c, 6a, c, d, f, h; S2d, e, f, h; S3a, c, d; S4b-e; S6a, c-e, g; S7d; S8b, c and S9-c are provided with the paper. Data that support the findings of this study have been deposited in Gene Expression Omnibus (GEO) with the accession number GSE127815.

Received: 13 September 2018 Accepted: 11 March 2019

Published online: 12 April 2019

\section{References}

1. Kempermann, G., Song, H. \& Gage, F. H. Neurogenesis in the adult hippocampus. Cold Spring Harb. Perspect. Biol. 7, a018812 (2015).

2. Lim, D. A. \& Alvarez-Buylla, A. Adult neural stem cells stake their ground. Trends Neurosci. 37, 563-571 (2014)

3. Lim, D. A. \& Alvarez-Buylla, A. The adult ventricular-subventricular zone (V-SVZ) and olfactory bulb (OB) neurogenesis. Cold Spring Harb. Perspect. Biol. 8, 1-33 (2016).

4. Merkle, F. T., Tramontin, A. D., Garcia-Verdugo, J. M. \& Alvarez-Buylla, A. Radial glia give rise to adult neural stem cells in the subventricular zone. Proc. Natl Acad. Sci. USA 101, 17528-17532 (2004).

5. Suh, H. et al. In vivo fate analysis reveals the multipotent and self-renewal capacities of Sox $2+$ neural stem cells in the adult hippocampus. Cell Stem Cell 1, 515-528 (2007).

6. Tavazoie, M. et al. A specialized vascular niche for adult neural stem cells. Cell Stem Cell 3, 279-288 (2008).

7. Doetsch, F., Garcia-Verdugo, J. M. \& Alvarez-Buylla, A. Cellular composition and three-dimensional organization of the subventricular germinal zone in the adult mammalian brain. J. Neurosci. 17, 5046-5061 (1997).

8. Mirzadeh, Z., Doetsch, F., Sawamoto, K., Wichterle, H. \& Alvarez-Buylla, A. The subventricular zone en-face: wholemount staining and ependymal flow. J. Vis. Exp. 39, 1-8 (2010).

9. Chaker, Z., Codega, P. \& Doetsch, F. A mosaic world: puzzles revealed by adult neural stem cell heterogeneity. Wiley Interdiscip Rev. Dev. Bio. 5, 640-658 (2016).

10. Bond, A. M., Ming, G. L. \& Song, H. Adult mammalian neural stem cells and neurogenesis: five decades later. Cell Stem. Cell. 17, 385-395 (2015).

11. Gonzalez-Perez, O., Romero-Rodriguez, R., Soriano-Navarro, M., GarciaVerdugo, J. M. \& Alvarez-Buylla, A. Epidermal growth factor induces the progeny of subventricular zone type B cells to migrate and differentiate into oligodendrocytes. Stem Cells 27, 2032-2043 (2009)

12. Menn, B. et al. Origin of oligodendrocytes in the subventricular zone of the adult brain. J Neurosci 26, 7907-7918 (2006).

13. Ferguson-Smith, A. C. Genomic imprinting: the emergence of an epigenetic paradigm. Nat. Rev. Genet. 12, 565-575 (2011).

14. Cleaton, M. A., Edwards, C. A. \& Ferguson-Smith, A. C. Phenotypic outcomes of imprinted gene models in mice: elucidation of pre- and postnatal functions of imprinted genes. Annu. Rev. Genomics Hum. Genet. 15, 93-126 (2014).

15. Wilkinson, L. S., Davies, W. \& Isles, A. R. Genomic imprinting effects on brain development and function. Nat. Rev. Neurosci. 8, 832-843 (2007).

16. Ferron, S. R. et al. Postnatal loss of Dlk1 imprinting in stem cells and niche astrocytes regulates neurogenesis. Nature 475, 381-385 (2011).

17. Ferron, S. R. et al. Differential genomic imprinting regulates paracrine and autocrine roles of IGF2 in mouse adult neurogenesis. Nat. Commun. 6, 8265 (2015).

18. Kar, S. et al. Epigenetic choreography of stem cells: the DNA demethylation episode of development. Cell. Mol. Life Sci. 71, 1017-1032 (2014).

19. Lehtinen, M. K. et al. The cerebrospinal fluid provides a proliferative niche for neural progenitor cells. Neuron 69, 893-905 (2011).

20. Edwards, C. A. \& Ferguson-Smith, A. C. Mechanisms regulating imprinted genes in clusters. Curr. Opin. Cell Biol. 19, 281-289 (2007).

21. Bird, A. Perceptions of epigenetics. Nature 447, 396-398 (2007).

22. Yao, B. et al. Epigenetic mechanisms in neurogenesis. Nat. Rev. Neurosci. 17, 537-49 (2016).

23. Wu, X. \& Zhang, Y. TET-mediated active DNA demethylation: mechanism, function and beyond. Nat. Rev. Genet. 18, 517-534 (2017).

24. Ito, S. et al. Tet proteins can convert 5-methylcytosine to 5-formylcytosine and 5-carboxylcytosine. Science 333, 1300-1303 (2011).

25. Tahiliani, M. et al. Conversion of 5-methylcytosine to 5hydroxymethylcytosine in mammalian DNA by MLL partner TET1. Science 324, 930-935 (2009).

26. Dawlaty, M. M. et al. Loss of Tet enzymes compromises proper differentiation of embryonic stem cells. Dev. Cell. 29, 102-111 (2014).

27. Hahn, M. A. et al. Dynamics of 5-hydroxymethylcytosine and chromatin marks in Mammalian neurogenesis. Cell reports 3, 291-300 (2013).

28. $\mathrm{Li}, \mathrm{X}$. et al. Ten-eleven translocation 2 interacts with forkhead box $\mathrm{O} 3$ and regulates adult neurogenesis. Nat. Commun. 8, 15903 (2017).

29. Zhang, R. R. et al. Tetl regulates adult hippocampal neurogenesis and cognition. Cell. Stem. Cell. 13, 237-245 (2013).

30. Li, T. et al. Critical role of Tet3 in neural progenitor cell maintenance and terminal differentiation. Mol. Neurobiol. 51, 142-154 (2015).

31. Hackett, J. A. et al. Germline DNA demethylation dynamics and imprint erasure through 5-hydroxymethylcytosine. Science 339, 448-452 (2013).

32. Liu, L. et al. Differential regulation of genomic imprinting by TET proteins in embryonic stem cells. Stem Cell Res. 15, 435-443 (2015).

33. Yamaguchi, S., Shen, L., Liu, Y., Sendler, D. \& Zhang, Y. Role of Tet1 in erasure of genomic imprinting. Nature 504, 460-464 (2013).

34. Chen, Q., Chen, Y., Bian, C., Fujiki, R. \& Yu, X. TET2 promotes histone O-GlcNAcylation during gene transcription. Nature 493, 561-564 (2013).

35. Vella, P. et al. Tet proteins connect the O-linked $\mathrm{N}$-acetylglucosamine transferase Ogt to chromatin in embryonic stem cells. Mol. Cell 49, 645-656 (2013).

36. Lian, H., Li, W. B. \& Jin, W. L. The emerging insights into catalytic or noncatalytic roles of TET proteins in tumors and neural development. Oncotarget 7, 64512-64525 (2016).

37. Montagner, S. et al. TET2 regulates mast cell differentiation and proliferation through catalytic and non-catalytic activities. Cell Rep. 20, 1744 (2017).

38. Kaas, G. A. et al. TET1 controls CNS 5-methylcytosine hydroxylation, active DNA demethylation, gene transcription, and memory formation. Neuron $\mathbf{7 9}$, 1086-1093 (2013).

39. Jin, S. G., Wu, X., Li, A. X. \& Pfeifer, G. P. Genomic mapping of 5hydroxymethylcytosine in the human brain. Nucleic Acids Res. 39, 5015-5024 (2011).

40. Munzel, M. et al. Quantification of the sixth DNA base hydroxymethylcytosine in the brain. Angew. Chem. Int. Ed. Engl. 49, 5375-5377 (2010).

41. Szulwach, K. E. et al. 5-hmC-mediated epigenetic dynamics during postnatal neurodevelopment and aging. Nat. Neurosci. 14, 1607-1616 (2011).

42. Peat, J. R. et al. Genome-wide bisulfite sequencing in zygotes identifies demethylation targets and maps the contribution of TET3 oxidation. Cell Rep. 9, 1990-2000 (2014).

43. Santos, F. et al. Active demethylation in mouse zygotes involves cytosine deamination and base excision repair. Epigenetics Chromatin 6, 39 (2013).

44. Garcia, A. D., Doan, N. B., Imura, T., Bush, T. G. \& Sofroniew, M. V. GFAPexpressing progenitors are the principal source of constitutive neurogenesis in adult mouse forebrain. Nat. Neurosci. 7, 1233-1241 (2004).

45. Ferron, S. R. et al. A combined ex/in vivo assay to detect effects of exogenously added factors in neural stem cells. Nat. Protoc. 2, 849-859 (2007).

46. Luo, J., Daniels, S. B., Lennington, J. B., Notti, R. Q. \& Conover, J. C. The aging neurogenic subventricular zone. Aging. Cell 5, 139-152 (2006).

47. Reynolds, B. A. \& Weiss, S. Generation of neurons and astrocytes from isolated cells of the adult mammalian central nervous system. Science $\mathbf{2 5 5}$, $1707-1710$ (1992)

48. Belenguer, G., Domingo-Muelas, A., Ferron, S. R., Morante-Redolat, J. M. \& Farinas, I. Isolation, culture and analysis of adult subependymal neural stem cells. Differentiation 91, 28-41 (2016). 
49. Costa, M. R. et al. Continuous live imaging of adult neural stem cell division and lineage progression in vitro. Development 138, 1057-1068 (2011).

50. Raponi, E. et al. S100B expression defines a state in which GFAP-expressing cells lose their neural stem cell potential and acquire a more mature developmental stage. Glia 55, 165-177 (2007).

51. Rraklli, V., Sodersten, E., Nyman, U., Hagey, D. W. \& Holmberg, J. Elevated levels of ZAC1 disrupt neurogenesis and promote rapid in vivo reprogramming. Stem Cell Res. 16, 1-9 (2016).

52. Gross, R. E. et al. Bone morphogenetic proteins promote astroglial lineage commitment by mammalian subventricular zone progenitor cells. Neuron 17 , 595-606 (1996).

53. Bond, A. M., Bhalala, O. G. \& Kessler, J. A. The dynamic role of bone morphogenetic proteins in neural stem cell fate and maturation. Dev. Neurobiol. 72, 1068-1084 (2012).

54. Korchynskyi, O. \& ten Dijke, P. Identification and functional characterization of distinct critically important bone morphogenetic protein-specific response elements in the Id1 promoter. J. Biol. Chem. 277, 4883-4891 (2002).

55. Bervini, S. \& Herzog, H. Mouse models of Prader-Willi Syndrome: a systematic review. Front. Neuroendocrinol. 34, 107-119 (2013).

56. Miyazaki, K. et al. Developmentally dynamic changes of DNA methylation in the mouse Snurf/Snrpn gene. Gene 432, 97-101 (2009).

57. Nicholls, R. D. \& Knepper, J. L. Genome organization, function, and imprinting in Prader-Willi and Angelman syndromes. Annu. Rev. Genomics Hum. Genet. 2, 153-175 (2001).

58. Jin, S. G. et al. Tet3 reads 5-Carboxylcytosine through Its CXXC domain and is a potential guardian against neurodegeneration. Cell. Rep. 14, 493-505 (2016).

59. Buiting, K. Prader-Willi syndrome and Angelman syndrome. Am. J. Med. Genet. C. Semin. Med. Genet. 154C, 365-376 (2010).

60. Butler, M. G. Prader-Willi syndrome: obesity due to genomic imprinting. Curr. Genomics 12, 204-215 (2011).

61. Li, H. et al. The autism-related gene SNRPN regulates cortical and spine development via controlling nuclear receptor Nr4al. Sci. Rep. 6, 29878 (2016).

62. Gomes, W. A., Mehler, M. F. \& Kessler, J. A. Transgenic overexpression of BMP4 increases astroglial and decreases oligodendroglial lineage commitment. Dev. Biol. 255, 164-177 (2003).

63. Lim, D. A. et al. Noggin antagonizes BMP signaling to create a niche for adult neurogenesis. Neuron 28, 713-726 (2000).

64. Guan, W. et al. Methylcytosine dioxygenase TET3 interacts with thyroid hormone nuclear receptors and stabilizes their association to chromatin. Proc. Natl Acad. Sci. USA 114, 8229-8234 (2017).

\section{Acknowledgements}

We firstly would like to thank Dr. Isabel Fariñas for reviewing and editing the manuscript and her group for technical support and discussion of the data. We thank Elba Barberá for technical support with mice maintenance. We also thank Dr. Strogantsev for discussion of the data and Dr. Julian Peat for providing TET3 and TET3-CD mutated expression plasmids. This work was supported by grants from Ministerio de Economía y Competitividad (SAF2012-40107 and SAF2016-78845-R), Generalitat Valenciana (ACOMP2014-258), and Fundación BBVA to S.R.F. S.R.F. is a Ramón y Cajal investigator. R.M.-L. is funded by a Spanish FPI fellowship program and A.L.-U. is funded by the Generalitat Valenciana fellowship program. Work in the AFS lab was funded by grants from the MRC and Wellcome Trust.

\section{Author contribution}

R.M.-L., A.L.-U. and S.R.F. performed most of the experiments. M.I. helped with methylation studies. C.K. contributed to Tet 3 variants overexpression experiments. S.R.F. initiated, designed, and led the study and wrote the manuscript. R.M.-L., A.L.-U, A.C.F.-S. and W.R. contributed to experimental design, data analysis, discussion, and writing of the paper.

\section{Additional information}

Supplementary Information accompanies this paper at https://doi.org/10.1038/s41467019-09665-1.

Competing interests: The authors declare no competing interests.

Reprints and permission information is available online at http://npg.nature.com/ reprintsandpermissions/

Journal Peer Review Information: Nature Communications thanks the anonymous reviewer(s) for their contribution to the peer review of this work. Peer reviewer reports are available.

Publisher's note: Springer Nature remains neutral with regard to jurisdictional claims in published maps and institutional affiliations.

(c) (i) Open Access This article is licensed under a Creative Commons Attribution 4.0 International License, which permits use, sharing, adaptation, distribution and reproduction in any medium or format, as long as you give appropriate credit to the original author(s) and the source, provide a link to the Creative Commons license, and indicate if changes were made. The images or other third party material in this article are included in the article's Creative Commons license, unless indicated otherwise in a credit line to the material. If material is not included in the article's Creative Commons license and your intended use is not permitted by statutory regulation or exceeds the permitted use, you will need to obtain permission directly from the copyright holder. To view a copy of this license, visit http://creativecommons.org/ licenses/by/4.0/.

(C) The Author(s) 2019 